Solutions of affine stochastic functional differential equations in the state space

Riedle, Markus

2007

MIMS EPrint: 2007.165

Manchester Institute for Mathematical Sciences

School of Mathematics

The University of Manchester

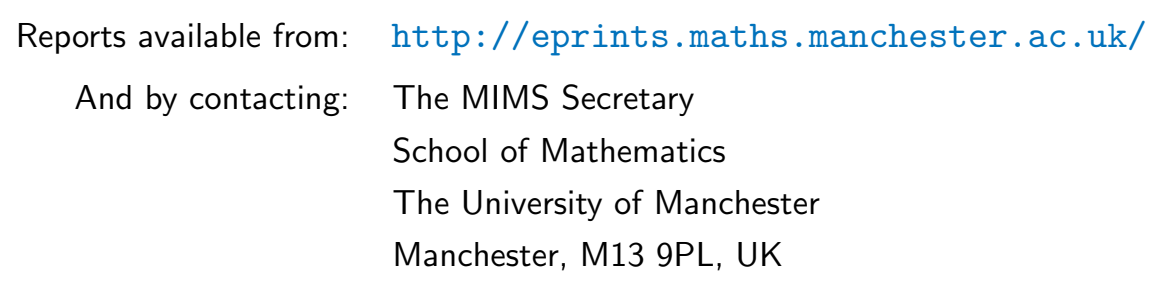

ISSN 1749-9097 


\title{
Solutions of affine stochastic functional differential equations in the state space
}

\author{
M. Riedle* \\ Humboldt-University of Berlin \\ Institute of Mathematics \\ 10099 Berlin, Germany
}

10 March 2005

\begin{abstract}
In this article we consider solutions of affine stochastic functional differential equations on $\mathbb{R}^{d}$. The drift of these equations is specified by a functional defined on a general function space $\mathcal{B}$ which is only described axiomatically. The solutions are reformulated as stochastic processes in the space $\mathcal{B}$. By representing such a process in the bidual space of $\mathcal{B}$ we establish that the transition functions of this process form a generalized Gaussian Mehler semigroup on $\mathcal{B}$. Thus the process is characterized completely on $\mathcal{B}$ since it is Markovian.

Moreover we derive a sufficient and necessary condition on the underlying space $\mathcal{B}$ such that the transition functions are even an Ornstein-Uhlenbeck semigroup. We exploit this result to associate a Cauchy problem in the function space $\mathcal{B}$ to the finite-dimensional functional equation.
\end{abstract}

\section{Introduction}

In this article we consider affine stochastic functional differential equations. Various approaches on functional differential equations exploit the idea of associating an equation in a function space to the functional equation under consideration. By this procedure which we call lifting the dependency on the past is removed. As we will see below a canonical choice of this function space is a specific instance of an $L^{p}$ space. In a deterministic setting the lifting to such an $L^{p}$ space was used for instance in [4] and related work by the same authors when dealing with problems in viscoelasticity, in [13], [14] and [21] for control problems or [8] for some general aspects. Stochastic equations are lifted to the same $L^{p}$ space with $p=2$ in [3]. More recently in [6], this approach was applied to obtain a stochastic evolution equation in a Hilbert space, which we present in the example below:

Example 1.1. The drift of an affine stochastic differential equation with delay is described by a linear functional on some function space. For $\alpha<0$ let $C([\alpha, 0])$ denote the space of continuous functions on the interval $[\alpha, 0]$ equipped with the

\footnotetext{
*riedle@mathematik.hu-berlin.de
} 
supremum norm. By choosing as most often in the literature the space $C([\alpha, 0])$ as the domain of the linear functional, we arrive at:

$$
\begin{aligned}
d X(t) & =\left(\int_{[\alpha, 0]} X(t+s) \mu(d s)\right) d t+d W(t) \quad \text { for } t \geqslant 0, \\
X(u) & =\varphi(u) \quad \text { for } u \in[\alpha, 0],
\end{aligned}
$$

where $\mu$ is a finite signed measure, $W$ is a real-valued Wiener process and the initial data $\varphi$ is in $C([\alpha, 0])$. For the underlying deterministic differential equation

$$
\begin{aligned}
\dot{x}(t) & =\int_{[\alpha, 0]} x(t+s) \mu(d s) \quad \text { for almost all } t \geqslant 0, \\
x(u) & =\varphi(u) \quad \text { for } u \in[\alpha, 0],
\end{aligned}
$$

there exists a unique solution $x(\cdot, \varphi)$ and the solution operators

$$
T(t): C([\alpha, 0]) \rightarrow C([\alpha, 0]), \quad(T(t) \varphi)(u)=x(t+u, \varphi),
$$

form a strongly continuous semigroup of bounded operators on $C([\alpha, 0])$, see [11]. Let $L_{\nu}^{2}$ denote the space of square-integrable functions on $[\alpha, 0]$ with respect to the measure $\nu(d t)=\delta_{0}(d t)+d t$ and equipped with the standard norm. The solution operators $T(t)$ can be extended to linear bounded operators on $L_{\nu}^{2}$ and the family $(T(t))_{t \geqslant 0}$ forms also a strongly continuous semigroup on $L_{\nu}^{2}$, see [7]. Denoting the generator of this semigroup by $A$ we can formulate a Cauchy problem on $L_{\nu}^{2}$ :

$$
\begin{aligned}
d Z(t) & =A Z(t) d t+G d W(t) \quad \text { for } t \geqslant 0, \\
Z(0) & =\varphi
\end{aligned}
$$

where the operator $G: \mathbb{R} \rightarrow L_{\nu}^{2}$ is defined by $G(s):=s \mathbb{1}_{\{0\}}(\cdot)$ for $s \in \mathbb{R}$. In [3] it is shown that the evaluation $Z(t)(0)$ of the weak solution $Z$ of (1.2) satisfies (1.1), and vice versa, if $X$ is the solution of (1.1) and if $X_{t}$ denotes the function $u \mapsto X(t+u)$ for $u \in[\alpha, 0]$, the $L_{\nu}^{2}$-valued segment process $\left(X_{t}: t \geqslant 0\right)$ is a weak solution of equation (1.2).

In the example the solution of (1.2) and consequently the segment process $\left(X_{t}\right.$ : $t \geqslant 0)$ is an Ornstein-Uhlenbeck process in the enlarged space $L_{\nu}^{2}$ and thus well analyzed. However, much less is known for the segment process on the original space $C([\alpha, 0])$. But for instance when dealing with problems in control or stability theory results are desired in the topology of the original space without enlarging it. Moreover, the lifting in the example depends strongly on the space $C([\alpha, 0])$ and the representation of the linear drift functional. It does not allow to conclude what happens if the original space differs from $C([\alpha, 0])$ which may occur as in applications the problem under consideration determines the function space. The example raises two questions:

- how can we characterize the segment process in the original function space without enlarging it, in particular for arbitrary function spaces? 
- which function spaces imply that the segment process solves an abstract Cauchy problem on them?

In order to deal with these questions we provide a general formulation of the problem where we do not specify the original function space, say $\mathcal{B}$. We include equations with infinite delay by assuming $\mathcal{B} \subseteq\left\{\varphi:(-\infty, 0] \rightarrow \mathbb{R}^{d}\right\}$. Equations depending only on functions with compact support such as in the example will be embedded later in this setting. The space $\mathcal{B}$ will be equipped with a seminorm $\|\cdot\|_{\mathcal{B}}$ and endowed with the induced topology. For a function $x: \mathbb{R} \rightarrow \mathbb{R}^{d}$ the segment function $x_{t}$ denotes the shifted function $x_{t}:(-\infty, 0] \rightarrow \mathbb{R}^{d}$ with $x_{t}(u):=x(t+u)$ for $u \leqslant 0$. Let $(\Omega, \mathscr{F}, P)$ be a probability space equipped with a filtration $\left\{\mathscr{F}_{t}\right\}_{t \geqslant 0}$. In this article we deal with the following affine stochastic differential equation with delay:

$$
\begin{aligned}
d X(t) & =L\left(X_{t}\right) d t+d W(t) \quad \text { for } t \geqslant 0, \\
X(u) & =\varphi(u) \quad \text { for } u \leqslant 0,
\end{aligned}
$$

where $W$ is a Brownian motion with values in $\mathbb{R}^{d}$. The initial value $\varphi$ is in $\mathcal{B}$ and the functional $L: \mathcal{B} \rightarrow \mathbb{R}^{d}$ is linear and continuous on $\mathcal{B}$. A solution $(X(t, \varphi)$ : $t \in \mathbb{R})$ is an adapted stochastic process with continuous paths satisfying $P$-a.s.

$$
X(t)=\varphi(0)+\int_{0}^{t} L\left(X_{s}\right) d s+W(t) \text { for all } t \geqslant 0,
$$

with $X_{0}=\varphi$. The solution is called unique if all solutions are indistinguishable. The segment process in $\mathcal{B}$ of a solution is denoted by $\left(X_{t}(\cdot, \varphi): t \geqslant 0\right)$.

Given this setting we will answer the question above in the following way:

- the segment process $\left(X_{t}(\cdot, \varphi): t \geqslant 0\right)$ on $\mathcal{B}$ turns out to be a pathwise continuous, Gaussian, strong Markov process. Its transition functions form a generalized Gaussian Mehler semigroup, a terminology explained in the Appendix; in general this is not an Ornstein-Uhlenbeck semigroup.

- we derive sufficient and necessary conditions on the space $\mathcal{B}$ such that we can associate a stochastic evolution equation on $\mathcal{B}$ which in addition is equivalent to the fact that the transition semigroup is an Ornstein-Uhlenbeck semigroup.

These results allow to study the solution of (1.3) in the topology of the arbitrary space $\mathcal{B}$ by means of its transition functions.

We end this introduction with summarizing the article. In the next section we consider the underlying deterministic differential equation. The problems treated in this article require not to specify the function space $\mathcal{B}$ but not every space $\mathcal{B}$ allows to solve equation (1.3). We tackle this problem by an approach developed in the theory of deterministic differential equations with infinite delay. There the admissible spaces $\mathcal{B}$ are only described axiomatically by some conditions which we also introduce in the next section.

The linearity of the equation allows to derive easily a variation of constants formula for the segment process $\left(X_{t}(\cdot, \varphi): t \geqslant 0\right)$, which we present in the 
beginning of Section 3. Since the calculation of the transition functions fails using this formula, we continue to establish a more appropriate representation of the segment process in the larger bidual space $\mathcal{B}^{* *}$.

This representation in $\mathcal{B}^{* *}$ enables us to calculate the transition functions of the segment process in Section 4. It turns out that the transition functions form a generalized Gaussian Mehler semigroup. As a consequence of this result we obtain that the segment process $\left(X_{t}(\cdot, \varphi): t \geqslant 0\right)$ is a strong Markov process in $\mathcal{B}$.

In the last part we derive sufficient and necessary conditions on $\mathcal{B}$ such that the transition functions form even an Ornstein-Uhlenbeck semigroup on $\mathcal{B}$, not only a Gaussian Mehler semigroup. We relate this result to the fact that the segment process is the weak solution of a Cauchy problem on $\mathcal{B}$. For establishing this relation we evoke the theory of stochastic convolution integrals on Banach spaces, rather recently introduced in [2] and [20].

In the Appendix we summarize some definitions and results on Gaussian semigroups and on stochastic convolution integrals on Banach spaces.

\section{Linear Autonomous Systems}

In this section we collect several results on the underlying deterministic differential equation of the stochastic equation (1.3), mostly from [12]. A linear delay differential equation is of the following form:

$$
\dot{x}(t)=L\left(x_{t}\right) \quad \text { for almost every } t \geqslant 0, \quad x_{0}=\varphi \in \mathcal{B},
$$

where $L: \mathcal{B} \rightarrow \mathbb{R}^{d}$ is a linear bounded functional. We say, that a solution of (2.4) is a function $x=x(\cdot, \varphi)$ on $\mathbb{R}$ which is locally absolutely continuous on $[0, \infty)$ and satisfies the first equation in (2.4) with $x_{0}=\varphi$.

The space $\mathcal{B}=\mathcal{B}\left((-\infty, 0], \mathbb{R}^{d}\right)$ is always assumed to be a linear subspace of $\{\varphi$ : $\left.(-\infty, 0] \rightarrow \mathbb{R}^{d}\right\}$ with semi-norm $\|\cdot\|_{\mathcal{B}}$ and endowed with the induced topology. A norm on $\mathbb{R}^{d}$ is denoted by $|\cdot|$. We denote by $C\left(J, \mathbb{R}^{d}\right)$ the space of bounded continuous functions mapping an interval $J$ into $\mathbb{R}^{d}$ with the norm $\|f\|_{C(J)}:=$ $\sup \{|f(u)|: u \in J\}$.

In the sequel we summarize the conditions on $\mathcal{B}$ as they are proposed in [12].

Condition (A). For every function $x: \mathbb{R} \rightarrow \mathbb{R}^{d}$ which is continuous on $[0, \infty)$ and satisfies $x_{0} \in \mathcal{B}$ the following conditions hold for every $t \geqslant 0$ :

1) $x_{t} \in \mathcal{B}$

2) there exists $H>0$, independent of $x$ and $t$, such that $|x(t)| \leqslant H\left\|x_{t}\right\|_{\mathcal{B}}$;

3) there exists $N:[0, \infty) \rightarrow[0, \infty)$, continuous, independent of $x$ and $t$, there exists $M:[0, \infty) \rightarrow[0, \infty)$, locally bounded, independent of $x$ and $t$, such that:

$$
\left\|x_{t}\right\|_{\mathcal{B}} \leqslant N(t) \sup _{0 \leqslant u \leqslant t}|x(u)|+M(t)\left\|x_{0}\right\|_{\mathcal{B}} .
$$

4) $t \mapsto x_{t}$ is a $\mathcal{B}$-valued continuous function. 
A space $\mathcal{B}$ satisfying Condition (A) always contains the space $C_{c}\left(\mathbb{R}_{-}, \mathbb{R}^{d}\right)$, the space of continuous functions on $\mathbb{R}_{-}:=(-\infty, 0]$ with compact support. This is due to the fact, that every function $\varphi$ which is continuous on $[0, \infty)$ and vanishes on $(-\infty, 0]$ is a function considered in Condition (A).1.

The homogeneous equation (2.4) has a unique solution under Condition (A). Thus, for $t \geqslant 0$ we can define solution operators:

$$
T(t): \mathcal{B} \rightarrow \mathcal{B}, \quad T(t) \varphi=x_{t},
$$

where $x=x(\cdot, \varphi)$ is the unique solution of equation (2.4) for $\varphi \in \mathcal{B}$.

For $\varphi \in \mathcal{B}$ the symbol $\hat{\varphi}$ denotes the equivalence class $\left\{\psi \in \mathcal{B}:\|\psi-\varphi\|_{\mathcal{B}}=0\right\}$. The quotient space $\hat{\mathcal{B}}:=\mathcal{B} /\|\cdot\|_{\mathcal{B}}$ is a linear space with norm $\|\hat{\varphi}\|_{\hat{\mathcal{B}}}=\|\varphi\|_{\mathcal{B}}$. For a bounded linear operator $U$ on $\mathcal{B}$ let $\hat{U}$ be the induced operator $\hat{U} \hat{\varphi}=U \varphi$ for some $\varphi \in \hat{\varphi}$.

Condition (B). The quotient space $\hat{\mathcal{B}}$ is complete.

Condition (B) implies that the solution operators $(\hat{T}(t))_{t \geqslant 0}$ form a strongly continuous semigroup of bounded operators on $\hat{\mathcal{B}}$.

Condition (C). The space $\mathcal{B}$ is separable.

In the theory of deterministic equations with infinite delay Condition $(\mathrm{C})$ is often assumed when dealing with stability properties. In our context, Condition (C) implies that the $\sigma$-algebra induced by the cylindrical sets equals the Borel $\sigma$ algebra on $\mathcal{B}$.

In this paper we assume that the space $\mathcal{B}$ of initial functions satisfies the Conditions (A), (B) and (C). We call $\mathcal{B}$ phase space. Before we continue we present some examples of function spaces satisfying these conditions. For details and further examples we refer to [12].

Example 2.1. For a $\gamma \in \mathbb{R}$ we define the normed space

$$
\begin{aligned}
C_{\gamma}\left(\mathbb{R}_{-}, \mathbb{R}^{d}\right) & :=\left\{\varphi \in C\left(\mathbb{R}_{-}, \mathbb{R}^{d}\right): \lim _{u \rightarrow-\infty}|\varphi(u)| e^{-\gamma u} \text { exists in }[0, \infty)\right\}, \\
\|\varphi\|_{C_{\gamma}} & :=\sup _{u \leqslant 0}\left|\varphi(u) e^{-\gamma u}\right| .
\end{aligned}
$$

The space $C_{\gamma}\left(\mathbb{R}_{-}, \mathbb{R}^{d}\right)$ satisfies the Conditions (A), (B) and (C), where the functions $N$ and $M$ in Condition (A) can be chosen as

$$
N(t)=\max \left\{1, e^{\gamma t}\right\} \quad \text { and } \quad M(t)=e^{\gamma t} \text { for } t \geqslant 0 .
$$

Example 2.2. For $\alpha \leqslant 0$ and a nonnegative locally integrable function $g: \mathbb{R}_{-} \rightarrow$ $[0, \infty)$ and $p \geqslant 1$ define the semi-normed space

$$
\begin{aligned}
& \left(C[\alpha, 0] \times L_{g}^{p}\right)\left(\mathbb{R}_{-}, \mathbb{R}^{d}\right) \\
& \quad:=\left\{\varphi: \mathbb{R}_{-} \rightarrow \mathbb{R}^{d}: \varphi \text { is continuous on }[\alpha, 0], \int_{-\infty}^{\alpha}|\varphi(u)|^{p} g(u) d u<\infty\right\} \\
& \|\varphi\|_{C[\alpha, 0] \times L_{g}^{p}}:=\sup _{s \in[\alpha, 0]}|\varphi(s)|+\left(\int_{-\infty}^{\alpha}|\varphi(u)|^{p} g(u) d u\right)^{1 / p} .
\end{aligned}
$$


If there exists a locally bounded function $G: \mathbb{R}_{-} \rightarrow[0, \infty)$ such that $g(u+s) \leqslant$ $G(u) g(s)$ for every $u, s \leqslant \alpha$ then the space $\left(C[\alpha, 0] \times L_{g}^{p}\right)\left(\mathbb{R}_{-}, \mathbb{R}^{d}\right)$ satisfies the Conditions (A), (B) and (C). The functions $N$ and $M$ in Condition (A) can be chosen as

$$
N(t)=1+\left(\int_{-t}^{0} g(u) d u\right)^{1 / p}, \quad M(t)=\max \left\{\left(\int_{-t}^{0} g(u) d u\right)^{1 / p}, G(-t)^{1 / p}\right\}
$$

for $t \geqslant 0$. If $\alpha=0$, then the norm is simplified to

$$
\|\varphi\|_{\mathbb{R}^{d} \times L_{g}^{p}}:=|\varphi(0)|+\left(\int_{-\infty}^{0}|\varphi(u)|^{p} g(u) d u\right)^{1 / p}
$$

and we use the notation $\mathbb{R}^{d} \times L_{g}^{p}$.

Example 2.2 describes a space of initial functions for differential equations with infinite delay that often occurs in studies of mechanics of materials with memory, see [15] and the references therein. In addition, this example of a phase space enables us to deal also with finite delay equations in our frame work:

Example 2.3. A linear differential equation with finite delay on the space $C\left([\alpha, 0], \mathbb{R}^{d}\right)$ is of the form, cf. also Example 4.5:

$$
\dot{x}(t)=\int_{[\alpha, 0]} x(t+u) \mu(d u) \quad \text { for almost all } t \geqslant 0, \quad x_{0}=\psi
$$

for $\psi \in C\left([\alpha, 0], \mathbb{R}^{d}\right)$ and a finite signed measure $\mu$. By defining the function $g(u):=0$ for every $u<\alpha$ and

$$
L \varphi:=\int_{[\alpha, 0]} \varphi(u) \mu(d u) \quad \text { for } \varphi \in\left(C[\alpha, 0] \times L_{g}^{1}\right)\left(\mathbb{R}_{-}, \mathbb{R}^{d}\right),
$$

we obtain a linear bounded operator $L$ on $\left(C[\alpha, 0] \times L_{g}^{1}\right)\left(\mathbb{R}_{-}, \mathbb{R}^{d}\right)$. According to Example 2.2 this function space satisfies the Conditions (A), (B) and (C) and

$$
\dot{x}(t)=L\left(x_{t}\right) \quad \text { for a.e. } t \geqslant 0, \quad x_{0}(u)=\psi(u), u \in[\alpha, 0], \quad x_{0}(u)=0, u<\alpha,
$$

describes an equation in our setting of infinite delay equations whose solution is a solution of the equation with finite delay.

The space of functions of bounded variation on an interval $J \subseteq \mathbb{R}$ is denoted by $B V\left(J, \mathbb{R}^{d}\right)$ with norm $\|\cdot\|_{B V}$ and total variation $\operatorname{Var}[\cdot, J]$. We call a function $f \in B V\left((-\infty, 0], \mathbb{R}^{d}\right)$ normalized if it is left continuous on $(-\infty, 0)$ and $f(0)=0$. Based on the Riesz representation theorem one obtains the following result, cf. Theorem 3.4.2 in [12].

Theorem 2.4. For every linear bounded operator $L: \mathcal{B} \rightarrow \mathbb{R}^{d}$ there exists a unique function $\mu_{L}:(-\infty, 0] \rightarrow \mathbb{R}^{d \times d}$, locally of bounded variation and normalized with

$$
\begin{aligned}
& L \vartheta=\int d \mu_{L}(u) \vartheta(u) \quad \text { for all } \vartheta \in C_{c}\left(\mathbb{R}_{-}, \mathbb{R}^{d}\right), \\
& \operatorname{Var}\left[\mu_{L},\left[a_{1}, a_{2}\right]\right] \leqslant c\|L\|_{\mathcal{B} \rightarrow \mathbb{R}^{d}} N\left(a_{2}-a_{1}\right) M\left(-a_{2}\right) \text { for } a_{1}<a_{2} \leqslant 0,
\end{aligned}
$$

where $c$ is a constant depending on the norm of $\mathbb{R}^{d}$. 
The function $\mu_{L}$ given in Theorem 2.4 defines two differential equations which are closely connected to equation (2.4). The first one is the so-called fundamental equation of (2.4):

$$
\dot{r}(t)=\int_{[-t, 0]} d \mu_{L}(u) r(t+u) \text { for almost every } t \geqslant 0, \quad r(0)=\mathrm{Id},
$$

where Id denotes the identity matrix in $\mathbb{R}^{d \times d}$. Theorem 4.1.3 in [12] guarantees the existence of a unique locally absolutely continuous function $r: \mathbb{R}_{+} \rightarrow \mathbb{R}^{d \times d}$ called fundamental solution satisfying the equations in (2.7).

The second equation defined by $\mu_{L}$ is the formal adjoint equation of (2.4):

$$
y(s)+\int_{s}^{0} y(u) \mu_{L}(s-u) d u=b(s) \quad \text { for all } s \leqslant 0,
$$

where the forcing function $b: \mathbb{R}_{-} \rightarrow \mathbb{R}^{d *}$ is locally of bounded variation and $\mathbb{R}^{d *}$ denotes the space of $d$-dimensional row-vectors.

According to Theorem 4.1.4 in [12] equation (2.8) has a unique solution $y=y(\cdot, b)$, which is locally of bounded variation for $s \leqslant 0$ :

$$
\operatorname{Var}[y,[s, 0]] \leqslant \operatorname{Var}[b,[s, 0]]+\left(e^{-c s\|L\|_{\mathcal{B} \rightarrow \mathbb{R}^{d}}\|N\|_{C[0,-s]}}-1\right) \sup _{s \leqslant u \leqslant 0}|b(u)|
$$

with a constant $c>0$ depending on the norm of $\mathbb{R}^{d}$. If the forcing function $b$ is normalized, then so is the solution $y(\cdot, b)$. Furthermore, by Corollary 4.1 .7 in [12] the solution of (2.8) is given by

$$
y(s)=b(0) r(-s)-\int_{[s, 0]} d b(u) r(u-s) \quad \text { for all } s \leqslant 0,
$$

where $r$ is the solution of (2.7).

Let $\mathcal{B}^{*}$ and $\hat{\mathcal{B}}^{*}$ be the dual spaces of $\mathcal{B}$ and $\hat{\mathcal{B}}$, respectively, which are Banach spaces with the usual operator norms. We denote by $\left\langle\varphi^{*}, \psi\right\rangle$ the duality pairing of $\psi \in \mathcal{B}$ and $\varphi^{*} \in \mathcal{B}^{*}$. The space $\mathcal{B}^{*}$ can be identified with $\hat{\mathcal{B}}^{*}$ by the mapping $\varphi^{*} \mapsto \hat{\varphi}^{*}$ for $\varphi^{*} \in \mathcal{B}^{*}$, where $\hat{\varphi}^{*} \in \hat{\mathcal{B}}^{*}$ is defined by $\left\langle\hat{\varphi}^{*}, \hat{\psi}\right\rangle=\left\langle\varphi^{*}, \psi\right\rangle$ for every $\psi \in \mathcal{B}$. In the same way one can identify the adjoint operator $\hat{U}^{*}$ of $\hat{U}$ with the adjoint operator $U^{*}$ of $U$ for a bounded linear operator $U$ on $\mathcal{B}$.

Theorem 2.4 implies that for every $\varphi^{*} \in \mathcal{B}^{*}$ a unique $\widetilde{\varphi}^{*}: \mathbb{R}_{-} \rightarrow \mathbb{R}^{d *}$ exists which is locally of bounded variation and normalized such that

$$
\begin{aligned}
& \qquad\left\langle\varphi^{*}, \vartheta\right\rangle=\int d \widetilde{\varphi}^{*}(u) \vartheta(u) \quad \text { for every } \vartheta \in C_{c}\left(\mathbb{R}_{-}, \mathbb{R}^{d}\right), \\
& \text { and } \operatorname{Var}\left[\widetilde{\varphi}^{*},[-t, 0]\right] \leqslant c N(t)\left\|\varphi^{*}\right\|_{\mathcal{B}^{*}} \text { for } t \geqslant 0 .
\end{aligned}
$$

For $\varphi^{*} \in \mathcal{B}^{*}$ we will always use the notation $\widetilde{\varphi}^{*}$ or $\left[\varphi^{*}\right]^{\sim}$ for the transformation introduced above.

The solution $y$ of the adjoint equation (2.8) and the adjoint operators $\left(T^{*}(t)\right)_{t \geqslant 0}$ of the solution operators $(T(t))_{t \geqslant 0}$ are related in the following way:

$$
\left[T^{*}(t) \varphi^{*}\right]^{(}(0-)=y\left(-t, \widetilde{\varphi}^{*}\right) \text { for every } t>0 \text { and } \quad \varphi^{*} \in \mathcal{B}^{*} .
$$

A proof is given in Theorem 4.2.2 in [12]. 


\section{Representations of the solution}

We begin this section with deriving representations of the solution of (1.3) both in the state space $\mathbb{R}^{d}$ and in the phase space $\mathcal{B}$. These will be based on the following random function: denoting the fundamental solution of (2.7) by $r$ we define for $t \geqslant 0$ :

$$
I(t): \mathbb{R}_{-} \rightarrow \mathcal{B}, \quad I(t)(u):= \begin{cases}\int_{0}^{t+u} r_{t-s}(u) d W(s), & \text { for } u \in[-t, 0], \\ 0, & \text { for } u<-t,\end{cases}
$$

where the integral is the Itô integral, which can be also viewed by partial integration as a Lebesgue-Stieltjes integral. Since the function $I(t)$ is pathwise continuous and vanishes on $(-\infty,-t]$ it is $\mathcal{B}$-valued.

Theorem 3.1. For every $\varphi \in \mathcal{B}$ there exists a unique solution $(X(t, \varphi): t \in \mathbb{R})$ of (1.3). The solution obeys for every $t \geqslant 0$ :

$$
X_{t}(\cdot, \varphi)=T(t) \varphi+I(t)
$$

and $X_{t}(\cdot, \varphi)$ is a $\mathcal{B}$-valued random variable.

Proof. The uniqueness of the solution of (1.3) follows by uniqueness of the solution of the homogeneous equation $(2.4)$. Let $X_{t}(\cdot, \varphi)$ be defined by $(3.14)$ and define $X(t):=X_{t}(0, \varphi), t \geqslant 0$, and $X(u)=\varphi(u), u<0$. Similarly to the finite delay case in [?] we calculate by means of (2.5) and partial integration:

$$
\begin{aligned}
& X(t)-\varphi(0)-W(t)-\int_{0}^{t} L X_{s} d s \\
& =(T(t) \varphi)(0)+\int_{0}^{t} \dot{r}(t-s) W(s) d s-\varphi(0)-\int_{0}^{t} L(T(s) \varphi+I(s)) d s \\
& =\int_{0}^{t} \dot{r}(t-s) W(s) d s-\int_{0}^{t}\left(\int_{[-s, 0]} d \mu_{L}(u) W(s+u)\right) d s \\
& \quad-\int_{0}^{t}\left(\int_{[-s, 0]} d \mu_{L}(u)\left(\int_{0}^{s+u} \dot{r}(s-v+u) W(v) d v\right)\right) d s \\
& =\int_{0}^{t} \dot{r}(t-s) W(s) d s-\int_{0}^{t} \mu_{L}(s-t) W(s) d s \\
& \quad-\int_{0}^{t}\left(\int_{[v-t, 0]} d \mu_{L}(u)\left(\int_{v-u}^{t} \dot{r}(s-v+u) d s\right)\right) W(v) d v \\
& =\int_{0}^{t} \dot{r}(t-s) W(s) d s-\int_{0}^{t} \mu_{L}(s-t) W(s) d s \\
& \quad-\int_{0}^{t}\left(\int_{[v-t, 0]} d \mu_{L}(u)(r(t-v+u)-\mathrm{Id})\right) W(v) d v \\
& =0 .
\end{aligned}
$$

As the process $(X(t): t \in \mathbb{R})$ is adapted it is a solution of (1.3). 
The measurability of $X_{t}$ follows by Pettis's measurability theorem, since for every $\varphi^{*} \in \mathcal{B}^{*}$ Fubini's theorem implies by (2.11) and (2.10):

$$
\begin{aligned}
\left\langle\varphi^{*}, I(t)\right\rangle & =\int d\left[\widetilde{\varphi}^{*}(u)\right]\left(\int_{0}^{t+u} r_{t-s}(u) d W(s)\right) \\
& =\int_{0}^{t}\left(\int_{[s-t, 0]} d\left[\widetilde{\varphi}^{*}(u)\right] r_{t-s}(u)\right) d W(s) \\
& =-\int_{0}^{t} y\left(s-t, \widetilde{\varphi}^{*}\right) d W(s),
\end{aligned}
$$

where $y\left(\cdot, \widetilde{\varphi}^{*}\right)$ denotes the solution of the adjoint equation (2.8). The last term in $(3.15)$ is $\mathcal{F}_{t}$-measurable which entails the same for $X_{t}$.

Note, that the representation (3.14) of the $\mathcal{B}$-valued random variable $X_{t}$ is simply obtained by using straightforward the definition of the segment function $X_{t}(u)=$ $X(t+u)$ applied to the representation in $\mathbb{R}^{d}$, which in turns implies the naive definition of the integral $I(t)$. However, we will see that this definition of the $\mathcal{B}$-valued integral has some drawbacks when calculating the covariance operator of the random variable $I(t)$. We get around these problems by deriving a weak*integral in the bidual space $\mathcal{B}^{* *}$ which coincides with $I(t)$ upon identifying $\mathcal{B}$ as a subspace of $\mathcal{B}^{* *}$, but which has the desired properties due to the larger space $\mathcal{B}^{* *}$.

In a different context we introduced the weak*-integral already in [16] and applied it to represent the solution of a deterministic delay equation. To keep our exposition self-contained we present the weak ${ }^{*}$-integral here again. We introduce it on an arbitrary real Banach space $E$ with norm $\|\cdot\|_{E}$. Later we will set $E=\mathcal{B}^{*}$.

Definition 3.2. A function $f:[a, b] \rightarrow E^{*}$ is called weak*-integrable on $[a, b]$ (with respect to continuous functions) if

1) the function $t \mapsto\langle f(t), x\rangle$ is of bounded variation on $[a, b]$ for each $x \in E$;

2) the linear operator

$$
F: E \rightarrow B V([a, b], \mathbb{R}), \quad F(x)(s):=\langle f(s), x\rangle, \quad s \in[a, b],
$$

is continuous.

Lemma 3.3. Let $f:[a, b] \rightarrow E^{*}$ be a weak $k^{*}$-integrable function and $h \in C([a, b], \mathbb{R})$. Then there exists a unique element $x^{*} \in E^{*}$ such that

$$
\left\langle x^{*}, x\right\rangle=\int_{a}^{b}\langle f(s), x\rangle d h(s) \quad \text { for all } x \in E,
$$

where the integral is to be understood as a Lebesgue-Stieltjes integral.

Proof. Define the operator $F: E \rightarrow B V([a, b], \mathbb{R})$ as in Definition 3.2. Since the operator $F$ is continuous, one obtains

$$
\left|\int_{a}^{b}\langle f(s), x\rangle d h(s)\right| \leqslant 2\|h\|_{C[a, b]}\|F(x)\|_{B V[a, b]} \leqslant 2\|h\|_{C[a, b]}\|F\|_{E \rightarrow B V}\|x\|_{E} .
$$


Hence, the linear functional $x \mapsto \int_{a}^{b}\langle f(s), x\rangle d h(s)$ is bounded and is thus an element of $E^{*}$.

Lemma 3.3 allows to define a weak*-integral:

Definition 3.4. Let $f:[a, b] \rightarrow E^{*}$ be a weak $k^{*}$-integrable function and $h \in$ $C([a, b], \mathbb{R})$. We define the weak*-integral of $f$ with respect to $h$ by the functional

$$
* \int_{a}^{b} f(s) d h(s) \in E^{*}: \quad\left\langle * \int_{a}^{b} f(s) d h(s), x\right\rangle:=\int_{a}^{b}\langle f(s), x\rangle d h(s)
$$

for all $x \in E$.

Let $f_{i}:[a, b] \rightarrow E^{*}$ be functions for $i=1, \ldots, d$ which are weak*-integrable. For $f=\left(f_{1}, \cdots, f_{d}\right)$ and $h \in C\left([a, b], \mathbb{R}^{d}\right)$ the weak*-integral $* f f d h$ is defined component-wise.

In the following definition we introduce a function which will turn out to be crucial for characterizing the segment process in the function space $\mathcal{B}$.

Definition 3.5. We define the operator

$$
\Upsilon: \mathcal{B}^{*} \rightarrow \mathbb{R}^{d *}, \quad \Upsilon\left(\varphi^{*}\right):=\left\langle\varphi^{*}, \Upsilon\right\rangle:=-\widetilde{\varphi}^{*}(0-),
$$

where $\widetilde{\varphi}^{*}(0-)$ denotes the left-hand sided limit in 0 of $\widetilde{\varphi}$.

We denote by $\Upsilon_{k}$ the k-th component of $\Upsilon$ and infer from (2.12)

$$
\left|\Upsilon_{k}\left(\varphi^{*}\right)\right| \leqslant\left|\widetilde{\varphi}^{*}(0-)\right| \leqslant \operatorname{Var}\left[\widetilde{\varphi}^{*},[-1,0]\right] \leqslant c N(1)\left\|\varphi^{*}\right\|_{B^{*}},
$$

which implies $\Upsilon_{k} \in \mathcal{B}^{* *}$ for $k=1, \ldots, d$. We set $U \Upsilon:=\left(U \Upsilon_{1}, \ldots, U \Upsilon_{d}\right)$ for an operator $U$ on $\mathcal{B}^{* *}$.

The operator $\Upsilon$ formalize the relation (2.13) between the adjoint solution operators $T^{*}(t)$ and the solution of the adjoint equation (2.8), i.e.

$$
\left\langle T^{*}(t) \varphi^{*}, \Upsilon\right\rangle=-y\left(-t, \widetilde{\varphi}^{*}\right) \quad \text { for every } \varphi^{*} \in \mathcal{B}^{*},
$$

where $y\left(\cdot, \widetilde{\varphi}^{*}\right)$ denotes the solution of the adjoint equation (2.8).

We identify the space $\hat{\mathcal{B}}$ with the subspace of the second dual space $\mathcal{B}^{* *}$ in the usual manner. The dual pairing of $\varphi^{*} \in \mathcal{B}^{*}$ and $\varphi^{* *} \in \mathcal{B}^{* *}$ is denoted by $\left\langle\varphi^{*}, \varphi^{* *}\right\rangle$. Now we utilize a weak*-integral according to Definition 3.4 where we replace the Banach space $E$ by the dual space $\hat{\mathcal{B}}^{*}$. By use of this weak ${ }^{*}$-integral we represent the segment of the solution of equation (1.3) in the second dual space $\hat{\mathcal{B}}^{* *}$ :

Theorem 3.6. Let $(X(t, \varphi): t \in \mathbb{R})$ be the solution of (1.3). Then $T^{* *}(t-\cdot) \Upsilon_{k}$ : $[0, t] \rightarrow \mathcal{B}^{* *}$ is weak $k^{*}$-integrable for all $k=1, \cdots, d$ and we have for every $t \geqslant 0$

$$
\hat{X}_{t}(\cdot, \varphi)=\hat{T}(t) \hat{\varphi}+* \int_{0}^{t} T^{* *}(t-s) \Upsilon d W(s) \quad P \text {-a.s. in } \hat{\mathcal{B}}^{* *}
$$


Proof. For $\varphi^{*} \in \mathcal{B}^{*}$ we denote by $y^{k}=y^{k}\left(\cdot, \widetilde{\varphi}^{*}\right)$ the $k$-th component of the solution $y\left(\cdot, \widetilde{\varphi}^{*}\right)$ of the adjoint equation $(2.8)$. The relation (3.16) for the function $\Upsilon$ implies

$$
\left\langle\varphi^{*}, T^{* *}(t-s) \Upsilon_{k}\right\rangle=\left\langle T^{*}(t-s) \varphi^{*}, \Upsilon_{k}\right\rangle=-y^{k}\left(s-t, \widetilde{\varphi}^{*}\right), \quad s \in[0, t] .
$$

Consequently, the function $s \mapsto\left\langle\varphi^{*}, T^{* *}(t-s) \Upsilon_{k}\right\rangle$ is of bounded variation on $[0, t]$ since $y\left(\cdot, \widetilde{\varphi}^{*}\right)$ is of bounded variation. The inequalities $(2.9)$ and $(2.12)$ yield

$$
\begin{aligned}
\left\|\left\langle\varphi^{*}, T^{* *}(t-\cdot) \Upsilon_{k}\right\rangle\right\|_{B V[0, t]} & =\left\|y^{k}\left(\cdot-t, \widetilde{\varphi}^{*}\right)\right\|_{B V[0, t]} \\
& \leqslant\left\|y\left(\cdot, \widetilde{\varphi}^{*}\right)\right\|_{B V[-t, 0]} \\
& =\left|y\left(-t, \widetilde{\varphi}^{*}\right)\right|+\operatorname{Var}\left[y\left(\cdot, \widetilde{\varphi}^{*}\right),[-t, 0]\right] \\
& \leqslant 2\left(\operatorname{Var}\left[\widetilde{\varphi}^{*},[-t, 0]\right]+b(t) \sup _{-t \leqslant u \leqslant 0}\left|\widetilde{\varphi}^{*}(u)\right|\right) \\
& \leqslant 2(1+2 b(t)) \operatorname{Var}\left[\widetilde{\varphi}^{*},[-t, 0]\right] \\
& \leqslant 2(1+2 b(t)) c N(t)\left\|\varphi^{*}\right\|_{\mathcal{B}^{*}},
\end{aligned}
$$

with $b(t):=\left(e^{c t\|L\|_{\mathcal{B} \rightarrow \mathbb{R}^{d}}\|N\|_{C[0, t]}}-1\right)$ and a constant $c>0$ depending on the norm of $\mathbb{R}^{d}$. Hence, the function $s \mapsto T^{* *}(t-s) \Upsilon_{k}$ is weak*-integrable for $k=$ $1, \cdots, d$. Moreover, by means of (3.16) and (3.15) we infer

$$
\begin{aligned}
\left\langle\varphi^{*}, * \int_{0}^{t} T^{* *}(t-s) \Upsilon d W(s)\right\rangle & =\int_{0}^{t}\left\langle\varphi^{*}, T^{* *}(t-s) \Upsilon\right\rangle d W(s) \\
& =\int_{0}^{t}\left\langle T^{*}(t-s) \varphi^{*}, \Upsilon\right\rangle d W(s) \\
& =-\int_{0}^{t} y\left(s-t, \widetilde{\varphi}^{*}\right) d W(s) \\
& =\left\langle\varphi^{*}, I(t)\right\rangle .
\end{aligned}
$$

The assertion follows by the variation of constants formula (3.14).

Remark 3.7. Since the solution of (1.3) is unique we obtain by Theorems 3.1 and 3.6 for every $t \geqslant 0$

$$
\int_{0}^{t} r_{t-s} d W(s)=* \int_{0}^{t} T^{* *}(t-s) \Upsilon d W(s) \quad P \text {-a.s. }
$$

upon identifying $\mathcal{B}$ as a subspace of $\hat{\mathcal{B}}^{* *}$. Note, that both integrals are defined pathwise.

Enlarging $\mathcal{B}$ to the larger space $\mathcal{B}^{* *}$ has enabled us to represent the segment process by means of the weak*-integral. In contrast to the naively defined integral $I(t)$ the weak*-integral is an element of the same space as its integrand and behaves therefore in many aspects as ordinary well-known integrals. As a consequence, we can easily compute in the next section the transition functions of the random variable defined by this weak ${ }^{*}$-integral and obtain those of $I(t)$ by Remark 3.7. 


\section{The Segment process in the Banach space}

In the remaining part of the article we derive properties of the stochastic process $\left(\hat{X}_{t}(\cdot, \varphi): t \geqslant 0\right)$ on the Banach space $\hat{\mathcal{B}}$ which is induced by the segment process $\left(X_{t}(\cdot, \varphi): t \geqslant 0\right)$. In order to keep the notations simple we assume in the sequel that the phase space $\mathcal{B}$ is a normed space. In the general situation all of the following results remain true on the induced space $\hat{\mathcal{B}}$.

Later we will see that the covariance operator $R_{t}$ of $I(t)$ is of the form

$$
R_{t} \varphi^{*}=\int_{0}^{t} T^{* *}(s) \Upsilon^{*} \Upsilon T^{*}(s) \varphi^{*} d s \quad \text { for } \varphi^{*} \in \mathcal{B}^{*},
$$

where the integral is defined as a Pettis integral:

$$
\left\langle\psi^{*}, R_{t} \varphi^{*}\right\rangle:=\int_{0}^{t}\left\langle\psi^{*}, T^{* *}(s) \Upsilon^{*} \Upsilon T^{*}(s) \varphi^{*}\right\rangle d s \quad \text { for every } \psi^{*} \in \mathcal{B}^{*}
$$

Although the integrand of the Pettis integral maps into the bidual space $\mathcal{B}^{* *}$ we obtain in this way a well-defined operator $R_{t}$ with values in $\mathcal{B}$ :

Theorem 4.1. For all $\varphi^{*} \in \mathcal{B}^{*}$ and $t \geqslant 0$ there exists a unique element $R_{t} \varphi^{*} \in \mathcal{B}$ satisfying

$$
\left\langle\psi^{*}, R_{t} \varphi^{*}\right\rangle=\int_{0}^{t}\left\langle\psi^{*}, T^{* *}(s) \Upsilon^{*} \Upsilon T^{*}(s) \varphi^{*}\right\rangle d s \quad \text { for every } \psi^{*} \in \mathcal{B}^{*}
$$

In this way one obtains a linear bounded operator $R_{t}: \mathcal{B}^{*} \rightarrow \mathcal{B}$ which is positive and symmetric.

Proof. By use of relation (3.16) between the solution $y(\cdot, \cdot)$ of the adjoint equation (2.8) and the adjoint operators $T^{*}(\cdot)$ the integrand of $R_{t}$ equals

$$
\begin{aligned}
\left\langle\psi^{*}, T^{* *}(s) \Upsilon^{*} \Upsilon T^{*}(s) \varphi^{*}\right\rangle & =\left\langle\Upsilon T^{*}(s) \psi^{*}, \Upsilon T^{*}(s) \varphi^{*}\right\rangle_{\mathbb{R}^{d *}} \\
& =y\left(-s, \widetilde{\psi}^{*}\right) y^{T}\left(-s, \widetilde{\varphi}^{*}\right) .
\end{aligned}
$$

The estimate (3.18) in the proof of Theorem 3.6 guarantees the existence of the integral in (4.19) and verifies the boundness of the operator $R_{t}: \mathcal{B}^{*} \rightarrow \mathcal{B}^{* *}$. It remains to show that $R_{t}$ is $\mathcal{B}$-valued. By the Krein-Shmulyan theorem this holds if and only if $R_{t} \varphi^{*}$ is weak ${ }^{*}$-continuous for every $\varphi^{*} \in \mathcal{B}^{*}$.

Since $\mathcal{B}$ is separable the linear functional $R_{t} \varphi^{*}: \mathcal{B}^{*} \rightarrow \mathbb{R}$ is weak ${ }^{*}$-continuous if and only if $R_{t} \varphi^{*}$ is weak ${ }^{*}$-sequentially continuous, see e.g. Corollary 12.8 in [5]. Here as well in the sequel we will utilize this result.

Let $\left(\psi_{n}^{*}\right)_{n \in \mathbb{N}}$ be a sequence in $\mathcal{B}^{*}$ that weak ${ }^{*}$-converges to $\psi^{*} \in \mathcal{B}^{*}$. The representation (2.11) for $\psi_{n}^{*}$ implies for every $\vartheta \in C_{c}\left(\mathbb{R}_{-}, \mathbb{R}^{d}\right)$ :

$$
\lim _{n \rightarrow \infty} \int d\left[\widetilde{\psi}_{n}^{*}(u)\right] \vartheta(u)=\lim _{n \rightarrow \infty}\left\langle\psi_{n}^{*}, \vartheta\right\rangle=\left\langle\psi^{*}, \vartheta\right\rangle=\int d\left[\widetilde{\psi}^{*}(u)\right] \vartheta(u) .
$$


Let $y\left(\cdot, \widetilde{\psi}_{n}^{*}\right)$ be the solution of the adjoint equation (2.8) with forcing function $\widetilde{\psi}_{n}^{*}$. By use of the variation of constants formula $(2.10)$ for $y\left(\cdot, \widetilde{\psi}_{n}^{*}\right)$ and relation (4.20) Fubini's theorem implies

$$
\begin{aligned}
\left\langle\psi_{n}^{*}, R_{t} \varphi^{*}\right\rangle & =\int_{0}^{t} y\left(-s, \widetilde{\psi}_{n}^{*}\right) y^{T}\left(-s, \widetilde{\varphi}^{*}\right) d s \\
& =-\int_{0}^{t}\left(\int_{[-s, 0]} d\left[\widetilde{\psi}_{n}^{*}(u)\right] r(u+s)\right) y^{T}\left(-s, \widetilde{\varphi}^{*}\right) d s \\
& =-\int_{[-t, 0]} d\left[\widetilde{\psi}_{n}^{*}(u)\right]\left(\int_{-u}^{t} r(u+s) y^{T}\left(-s, \widetilde{\varphi}^{*}\right) d s\right) .
\end{aligned}
$$

Since the inner integral in the last line defines a continuous function in $u$ with compact support we obtain by use of (4.21)

$$
\lim _{n \rightarrow \infty}\left\langle\psi_{n}^{*}, R_{t} \varphi^{*}\right\rangle=-\int_{[-t, 0]} d\left[\widetilde{\psi}^{*}(u)\right]\left(\int_{-u}^{t} r(u+s) y^{T}\left(-s, \widetilde{\varphi}^{*}\right) d s\right) .
$$

Applying the same computations as in (4.22) we see that the right hand side of the above relation equals $\left\langle\psi^{*}, R_{t} \varphi^{*}\right\rangle$. Therefore, we have

$$
\lim _{n \rightarrow \infty}\left\langle\psi_{n}^{*}, R_{t} \varphi^{*}\right\rangle=\left\langle\psi^{*}, R_{t} \varphi^{*}\right\rangle,
$$

which shows that $R_{t} \varphi^{*}$ is weak*-continuous.

Since the operator $R_{t}$ is symmetric and positive there is at least a Gaussian cylindrical measure on the Borel- $\sigma$-field $\mathfrak{B}(\mathcal{B})$ with covariance operator $R_{t}$, see for example Proposition VI.3.3 in [18]. If this cylindrical measure is a measure the operator $R_{t}$ is called $\gamma$-radonifying. Theorem 3.1 indicates already that $I(t)$ is a $\mathcal{B}$-valued random variable and by means of the weak* representation one can identify $R_{t}$ as its covariance operator in the following Corollary:

Corollary 4.2. The random variable

$$
I(t)=\int_{0}^{t} r_{t-s} d W(s)
$$

is for every $t \geqslant 0$ a Gaussian random variable on $(\mathcal{B}, \mathfrak{B}(\mathcal{B}))$ with covariance operator $R_{t}$.

Proof. In (3.15) we obtained the representation

$$
\left\langle\varphi^{*}, I(t)\right\rangle=-\int_{0}^{t} y\left(s-t, \widetilde{\varphi}^{*}\right) d W(s) \quad \text { for every } \varphi^{*} \in \mathcal{B}^{*},
$$

where $y$ denotes the solution of the adjoint equation. Since the integral is an Itô integral with a deterministic integrand the random variable $\left\langle\varphi^{*}, I(t)\right\rangle$ is Gaussian, thus $I(t)$ is also Gaussian. 
By Remark 3.7 and Itô's isometry we compute for $\varphi^{*} \in \mathcal{B}^{*}$ :

$$
\begin{aligned}
\mathbb{E}\left[\left\langle\varphi^{*}, I(t)\right\rangle\right]^{2} & =\mathbb{E}\left[\left\langle\varphi^{*}, * \int_{0}^{t} T^{* *}(t-s) \Upsilon d W(s)\right\rangle\right]^{2} \\
& =\mathbb{E}\left[\int_{0}^{t}\left\langle\varphi^{*}, T^{* *}(t-s) \Upsilon\right\rangle d W(s)\right]^{2} \\
& =\mathbb{E}\left[\int_{0}^{t}\left\langle T^{*}(t-s) \varphi^{*}, \Upsilon\right\rangle d W(s)\right]^{2} \\
& =\int_{0}^{t}\left\langle\Upsilon T^{*}(s) \varphi^{*}, \Upsilon T^{*}(s) \varphi^{*}\right\rangle_{\mathbb{R}^{d *}} d s \\
& =\left\langle\varphi^{*}, R_{t} \varphi^{*}\right\rangle,
\end{aligned}
$$

which establishes $R_{t}$ as the covariance operator of $I(t)$.

Corollary 4.2 implies that the segment process $\left(X_{t}(\cdot, \varphi): t \geqslant 0\right)$ for non-random $\varphi \in \mathcal{B}$ is a Gaussian stochastic process on the Banach space $\mathcal{B}$. As an immediate consequence of the next theorem we will obtain, that this process is Markovian on $\mathcal{B}$. For that, we consider the transition functions defined by

$$
P(t): B_{b}(\mathcal{B}, \mathbb{R}) \rightarrow B_{b}(\mathcal{B}, \mathbb{R}), \quad(P(t) f)(\varphi):=\mathbb{E}\left[f\left(X_{t}(\cdot, \varphi)\right)\right],
$$

where $B_{b}(\mathcal{B}, \mathbb{R})$ denotes the set of real-valued, bounded, Borel-measurable functions on $\mathcal{B}$. The variation of constants formula (3.14) for the segment process yields a special integral representation of the operators $P(t)$. For such operators, if in addition they have the semigroup property on $B_{b}(\mathcal{B}, \mathbb{R})$, the notation generalized Gaussian Mehler semigroup is introduced in [1].

Theorem 4.3. The transition functions of the segment process $\left(X_{t}(\cdot, \varphi): t \geqslant 0\right)$ form a Gaussian Mehler semigroup $(P(t))_{t \geqslant 0}$ on $B_{b}(\mathcal{B}, \mathbb{R})$ defined by $(T(t))_{t \geqslant 0}$ and $\left(\mu_{t}\right)_{t \geqslant 0}$ :

$$
(P(t) f)(\varphi)=\int_{\mathcal{B}} f(T(t) \varphi+\psi) \mu_{t}(d \psi) \quad \text { for } f \in B_{b}(\mathcal{B}, \mathbb{R}), \varphi \in \mathcal{B},
$$

where $\mu_{t}$ denotes the distribution of $I(t)$.

Proof. By the variation of constants formula (3.14) we compute

$$
\mathbb{E}\left[f\left(X_{t}(\cdot, \varphi)\right)\right]=\int_{\Omega} f(T(t) \varphi+I(t)(\omega)) P(d \omega)=\int_{\mathcal{B}} f(T(t) \varphi+\psi) \mu_{t}(d \psi) .
$$

Furthermore, from the identity

$$
R_{t+s}=T(s) R_{t} T^{*}(s)+R_{s} \quad \text { for every } s, t \geqslant 0,
$$

follows by means of the characteristical functions that

$$
\mu_{t+s}=T(s) \mu_{t} * \mu_{s} \quad \text { for every } s, t \geqslant 0,
$$

which implies that $(P(t))_{t \geqslant 0}$ is a Gaussian Mehler semigroup according to Definiton B.7. 
Theorem 4.3 establishes a new approach for studying the delay equation (1.3) by means of Gaussian Mehler semigroups. Since Gaussian Mehler semigroups behave in many aspects as Ornstein-Uhlenbeck semigroups one can evoke results on the latter to deal with the segment process.

Corollary 4.4. The segment process $\left(X_{t}(\cdot, \varphi): t \geqslant 0\right)$ is a Gaussian, strong Markov process on $(\mathcal{B}, \mathfrak{B}(\mathcal{B}))$ with continuous paths and transition functions as given in Theorem 4.3.

Proof. To establish the continuity we infer from the variaton of constants formula

$$
\left\|X_{t_{2}}(\cdot, \varphi)-X_{t_{1}}(\cdot, \varphi)\right\|_{\mathcal{B}} \leqslant\left\|T\left(t_{1}\right) \varphi-T\left(t_{2}\right) \varphi\right\|_{\mathcal{B}}+\left\|I\left(t_{1}\right)-I\left(t_{2}\right)\right\|_{\mathcal{B}}
$$

for $t_{2} \geqslant t_{1} \geqslant 0$. Due to Condition (A).3 on $\mathcal{B}$ we obtain

$$
\left\|I\left(t_{1}\right)-I\left(t_{2}\right)\right\|_{\mathcal{B}} \leqslant C_{t_{2}} \sup _{u \in\left[-t_{2}, 0\right]}\left|I\left(t_{2}\right)(u)-I\left(t_{1}\right)(u)\right|
$$

for a constant $C_{t_{2}}$ depending only on $t_{2}$. Applying partial integration to $I\left(t_{2}\right)(u)-$ $I\left(t_{1}\right)(u)$ some tedious calculations show that the right hand side tends to 0 as $t_{2}-t_{1} \rightarrow 0$ which is the continuity.

Since the transition semigroup is Feller, as it can be easily seen by the representation according to Theorem 4.3, the segment process is a strong Markov process by $[9$, Thm. 3.10].

Example 4.5. The segment process $\left(X_{t}(\cdot, \varphi): t \geqslant 0\right)$ of the solution of the equation (1.1) is a Gaussian, strong Markov process on the space $C([\alpha, 0])$ of continuous functions. Its transition functions are given by

$$
\mathbb{E}\left[f\left(X_{t}(\cdot, \varphi)\right)\right]=\int_{C[\alpha, 0]} f(T(t) \varphi+\psi) \mu_{t}(d \psi)
$$

for every bounded, measurable function $f: C([-\alpha, 0]) \rightarrow \mathbb{R}$, where $\mu_{t}$ is a Gaussian measure on $C([\alpha, 0])$ with covariance operator $R_{t}$.

Let us remark at this point that we deal in detail with this example in [19]. There we introduce a general weak* integral in locally convex spaces to obtain a representation of the segment process as in Theorem 3.6.

\section{The case of Ornstein-Uhlenbeck semigroup}

In order that the transition semigroup $(P(t))_{t \geqslant 0}$ forms an Ornstein-Uhlenbeck semigroup the covariance operators of the Gaussian measures $\mu_{t}$ have to be given by

$$
Q_{t}=\int_{0}^{t} T(s) Q Q^{*} T^{*}(s) d s
$$

for a linear bounded operator $Q \in \mathcal{L}\left(\mathbb{R}^{d}, \mathcal{B}\right)$. The operator $Q_{t}$ differs from the covariance operator $R_{t}$ defined in Theorem 4.1 only by the integrands which are in general $\mathcal{B}^{* *}$-valued for $R_{t}$. 
Theorem 5.1. Let $(X(t, \varphi): t \in \mathbb{R})$ be the solution of equation (1.3) for nonrandom $\varphi \in \mathcal{B}$. Then the following statements are equivalent:

(1) there exists $Q \in \mathcal{L}\left(\mathbb{R}^{d}, \mathcal{B}\right)$ such that $\left(X_{t}(\cdot, \varphi): t \geqslant 0\right)$ is an OrnsteinUhlenbeck process defined by $(T(t))_{t \geqslant 0}$ and $Q$;

(2) the functionals $\Upsilon_{k}: \mathcal{B}^{*} \rightarrow \mathbb{R}$ are weak $k^{*}$ continuous for $k=1, \ldots, d$.

(3) the operators $\Upsilon_{k}^{*}: \mathbb{R} \rightarrow \mathcal{B}^{* *}$ are $\mathcal{B}$-valued for $k=1, \ldots, d$.

Proof. We prove the theorem by $(2) \Leftrightarrow(3)$ and $(3) \Rightarrow(1) \Rightarrow(2)$.

From relation $(2.13)$ follows that there exists $\varphi^{*} \in \mathcal{B}^{*}$ with $\widetilde{\varphi}^{*}(0-) \neq 0$. Then condition (2) is equivalent to the fact that $\Upsilon_{k}^{*} \Upsilon_{k} \varphi^{*} \in \mathcal{B}^{* *}$ is weak ${ }^{*}$-continuous for every $\varphi^{*} \in \mathcal{B}^{*}$. By the Krein-Shmulyan theorem the latter is equivalent to the fact that the operator $\Upsilon_{k}^{*} \Upsilon_{k}: \mathcal{B}^{*} \rightarrow \mathcal{B}^{* *}$ is $\mathcal{B}$-valued. The last property is equivalent to condition (3) since $\Upsilon_{k}$ is surjective which follows by linearity and the existence of a $\varphi^{*} \in \mathcal{B}^{*}$ with $\widetilde{\varphi}^{*}(0-) \neq 0$.

For the proof $(3) \Rightarrow(1)$ note that condition (3) implies that $\Upsilon^{*}$ is also $\mathcal{B}$-valued. Hence, the covariance operator $R_{t}$ of $\mu_{t}$ obeys

$$
R_{t}=\int_{0}^{t} T^{* *}(s) \Upsilon^{*} \Upsilon T^{*}(s) d s=\int_{0}^{t} T(s) \Upsilon^{*} \Upsilon T^{*}(s) d s .
$$

Therefore, the Mehler semigroup $(P(t))_{t \geqslant 0}$ is an Ornstein-Uhlenbeck semigroup defined by $(T(t))_{t \geqslant 0}$ and $\Upsilon^{*}$.

For establishing that Condition (1) implies (2) let

$$
Q_{t}=\int_{0}^{t} T(s) Q Q^{*} T^{*}(s) d s \quad \text { for } t \geqslant 0
$$

be the covariance operator of the Ornstein-Uhlenbeck process $\left(X_{t}(\cdot, \varphi): t \geqslant 0\right)$. According to Corollary 4.2 and Theorem 4.3 we have $R_{t}=Q_{t}$ for every $t \geqslant 0$. Thus, considering $Q Q^{*}$ as a $\mathcal{B}^{* *}$-valued operator yields for every $\psi^{*}$ and $\varphi^{*} \in \mathcal{B}^{*}$ :

$$
\int_{0}^{t}\left\langle T^{*}(s) \psi^{*},\left(Q Q^{*}-\Upsilon^{*} \Upsilon\right) T^{*}(s) \varphi^{*}\right\rangle d s=0 \quad \text { for every } t \geqslant 0,
$$

which results in

$$
\left\langle\psi^{*}, T(s) Q Q^{*} T^{*}(s) \varphi^{*}\right\rangle=\left\langle\psi^{*}, T^{* *}(s) \Upsilon^{*} \Upsilon T^{*}(s) \varphi^{*}\right\rangle \text { for Lebesgue a.e. } s \geqslant 0 .
$$

Let $\left(s_{m}\right)_{m \in \mathbb{N}} \subseteq[0,1]$ be a sequence which converges to 0 as $m \rightarrow \infty$ such that for every $m \in \mathbb{N}$ this relation is satisfied. We denote the canonical basis of $\mathbb{R}^{d}$ by $e_{1}, \ldots, e_{d}$. Since the mapping

$$
s \mapsto\left\langle Q^{*} T^{*}(s) \psi^{*}, e_{k}\right\rangle_{\mathbb{R}^{d}}=\left\langle\psi^{*}, T(s) Q e_{k}\right\rangle
$$

is continuous for $k=1, \ldots, d$, we obtain

$$
\begin{aligned}
\left\langle\psi^{*}, T\left(s_{m}\right) Q Q^{*} T^{*}\left(s_{m}\right) \varphi^{*}\right\rangle & =\sum_{k=1}^{d}\left\langle Q^{*} T^{*}\left(s_{m}\right) \psi^{*}, e_{k}\right\rangle_{\mathbb{R}^{d}}\left\langle Q^{*} T^{*}\left(s_{m}\right) \varphi^{*}, e_{k}\right\rangle_{\mathbb{R}^{d}} \\
& \rightarrow\left\langle Q^{*} \psi^{*}, Q^{*} \varphi^{*}\right\rangle_{\mathbb{R}^{d}} \quad \text { as } m \rightarrow 0
\end{aligned}
$$


On the other hand, by use of the adjoint equation (2.8) we have

$$
\lim _{m \rightarrow \infty} y\left(-s_{m}, \widetilde{\psi}^{*}\right)=\widetilde{\psi}^{*}(0-)=-\left\langle\psi^{*}, \Upsilon\right\rangle
$$

which results in

$$
\begin{aligned}
\lim _{m \rightarrow \infty}\left\langle\psi^{*}, T^{* *}\left(s_{m}\right) \Upsilon^{*} \Upsilon T^{*}\left(s_{m}\right) \varphi^{*}\right\rangle & =\lim _{m \rightarrow \infty} y\left(-s_{m}, \widetilde{\psi}^{*}\right) y^{T}\left(-s_{m}, \widetilde{\varphi}^{*}\right) \\
& =\left\langle\Upsilon \psi^{*}, \Upsilon \varphi^{*}\right\rangle_{\mathbb{R}^{d}}
\end{aligned}
$$

The equations (5.24) and (5.25) yield $Q Q^{*}=\Upsilon^{*} \Upsilon$ and consequently $\Upsilon^{*} \Upsilon$ is $\mathcal{B}$ valued. Let $\left(\psi_{n}^{*}\right)_{n \in \mathbb{N}}$ be a sequence which weak*-converges to $\psi^{*} \in \mathcal{B}^{*}$ and let $\varphi_{j}^{*} \in \mathcal{B}^{*}$ be such that the span of $\left\{\Upsilon \varphi_{j}^{*}: j=1, \ldots, d^{\prime}\right\}$ equals $\left\{\Upsilon \psi_{n}^{*}: n \in \mathbb{N}\right\} \subseteq$ $\mathbb{R}^{d *}$. Since $\Upsilon^{*} \Upsilon$ is now known to be $\mathcal{B}$-valued we obtain

$\lim _{n \rightarrow \infty}\left\langle\Upsilon \psi_{n}^{*}, \Upsilon \varphi_{j}^{*}\right\rangle_{\mathbb{R}^{d}}=\lim _{n \rightarrow \infty}\left\langle\psi_{n}^{*}, \Upsilon^{*} \Upsilon \varphi_{j}^{*}\right\rangle=\left\langle\Upsilon^{*} \psi^{*}, \Upsilon \varphi_{j}^{*}\right\rangle_{\mathbb{R}^{d}}$ for every $j=1, \ldots, d^{\prime}$,

which implies that $\Upsilon$ and therefore $\Upsilon_{k}$ are weak*-continuous.

For simplifying the conditions in Theorem 5.1 we assume in the sequel that the spaces $\mathcal{B}$ are Banach lattices. Even if this assumption may be not necessary for the following the condition suits the axiomatic description of the phase spaces and does not constitute a substantial limitation as it is satisfied by all spaces occuring in the literature and applications.

We denote the canonical partial ordering in the Euclidean space $\mathbb{R}^{d}$ by $u \leqslant v$ for $u, v \in \mathbb{R}^{d}$. The phase spaces $\mathcal{B}$ are equipped with the partial ordering

$$
\varphi \leqslant \psi \Leftrightarrow \varphi(u) \leqslant \psi(u) \text { for every } u \leqslant 0
$$

If $\mathcal{B}$ is a Banach lattice the positive cone $\{\varphi \in \mathcal{B}: \varphi \geqslant 0\}$ is closed, see Proposition II.5.2 in [17]. The elements in the positive cone are called positive. Moreover, the dual space $\mathcal{B}^{*}$ enjoys the property that every element $\varphi^{*} \in \mathcal{B}^{*}$ obeys $\varphi^{*}=\varphi_{+}^{*}-\varphi_{-}^{*}$ where $\varphi_{+}^{*}, \varphi_{-}^{*} \in \mathcal{B}^{*}$ are positive functionals, see Proposition II.5.5 in [17]. A functional $\varphi^{*} \in \mathcal{B}^{*}$ is called positive if $\left\langle\varphi^{*}, \psi\right\rangle \geqslant 0$ for every positive $\psi \in \mathcal{B}$. Recall, that $e_{1}, \cdots, e_{d}$ denotes the canonical orthonormal basis of $\mathbb{R}^{d}$.

Theorem 5.2. If $\mathcal{B}$ is a Banach lattice then the following conditions are equivalent:

(1) there exists $Q \in \mathcal{L}\left(\mathbb{R}^{d}, \mathcal{B}\right)$ such that $\left(X_{t}(\cdot, \varphi): t \geqslant 0\right)$ is an OrnsteinUhlenbeck process defined by $(T(t))_{t \geqslant 0}$ and $Q$;

(2) the functions $u \mapsto \mathbb{1}_{\{0\}}(u) e_{k}$ for $u \leqslant 0$ are elements of $\mathcal{B}$ for $k=1, \ldots, d$.

Proof. To prove the implication $(1) \Rightarrow(2)$ we consider a fixed $k$. By Theorem 5.1 condition (1) implies that the functional $\Upsilon_{k}$ is weak*-continuous. Hence, there exists a $\varphi_{0} \in \mathcal{B}$ such that $\Upsilon_{k}\left(\psi^{*}\right)=\psi^{*}\left(\varphi_{0}\right)$ for every $\psi^{*} \in \mathcal{B}^{*}$ and it remains to show that $\varphi_{0}=\mathbb{1}_{\{0\}} e_{k}$. 
We define the functions $\vartheta_{n}(u):=\mathbb{1}_{[-1 / n, 0]}(1+n u) e_{k}$ for $u \leqslant 0$. By the representation (2.11) for functions in $C_{c}\left(\mathbb{R}_{-}, \mathbb{R}^{d}\right)$ and by partial integration we derive for every $\varphi^{*} \in \mathcal{B}^{*}$ :

$$
\begin{aligned}
\left\langle\varphi^{*}, \vartheta_{n}\right\rangle & =\int_{[-1 / n, 0]} d\left[\widetilde{\varphi}^{*}(u)\right] \vartheta_{n}(u) \\
& =-n \int_{-1 / n}^{0} \widetilde{\varphi}^{*}(u) e_{k} d u \rightarrow-\widetilde{\varphi}^{*}(0-) e_{k} \quad \text { as } n \rightarrow \infty .
\end{aligned}
$$

As the last term equals $\Upsilon_{k}\left(\varphi^{*}\right)$ the sequence $\left(\vartheta_{n}\right)_{n \geqslant 1}$ converges weakly to $\varphi_{0}$. By a corollary to the Hahn-Banach Theorem there is a convex combination $\theta_{n}$ of the $\left(\vartheta_{n}\right)_{n \in \mathbb{N}}$ such that $\left(\theta_{n}\right)_{n \in \mathbb{N}}$ converges strongly to $\varphi_{0}$. By choosing each $\theta_{n}$ in the span of $\vartheta_{n}, \vartheta_{n+1}, \ldots$ and passing, if necessary, to a subsequence we may furthermore arrange that $0 \leqslant \theta_{n} \leqslant \theta_{m}$ for every $n \geqslant m$. Therefore and since the cone $\{\varphi \in \mathcal{B}: \varphi \geqslant 0\}$ is closed we obtain $0 \leqslant \varphi_{0} \leqslant \theta_{n}$ for all $n \in \mathbb{N}$, which results in

$$
\varphi_{0}(u)=0 \text { for } u<0 \text { and } 0 \leqslant \varphi_{0}(0) \leqslant e_{k}=\theta_{n}(0) .
$$

Condition (A) guarantees that the evaluation functional $\pi^{*}: \mathcal{B} \rightarrow \mathbb{R}$ with $\pi^{*}(\varphi)=$ $\left\langle\varphi(0), e_{k}\right\rangle_{\mathbb{R}^{d}}$ for $\varphi \in \mathcal{B}$ is in $\mathcal{B}^{*}$. The weak convergence of $\left(\vartheta_{n}\right)_{n \in \mathbb{N}}$ implies

$$
1=\left\langle\pi^{*}, \vartheta_{n}\right\rangle \rightarrow\left\langle\pi^{*}, \varphi_{0}\right\rangle=\left\langle\varphi_{0}(0), e_{k}\right\rangle_{\mathbb{R}^{d}}
$$

and analogously $\left\langle\varphi_{0}(0), e_{l}\right\rangle_{\mathbb{R}^{d}}=0$ for $l \neq k$. Thus, we end with $\varphi_{0}(0)=e_{k}$ and the proof of (2) is complete.

We prove now the converse direction. Note, that if we define $r(u)=0$ for $u<0$ where $r$ denotes the fundamental solution then condition (2) implies, that the segments $r_{t} e_{k}$ are elements of the space $\mathcal{B}$ for every $t \geqslant 0$ and $k=1, \ldots, d$. We show first that the application of a functional $\varphi^{*} \in \mathcal{B}^{*}$ to $r_{t} e_{k}$ can be represented by an integral, see (5.27) below.

More general, by continuous continuation of the function $\mathbb{1}_{\{0\}} e_{k}$ condition (A) guarantees that for arbitrary $C>0$ every function $\varphi: \mathbb{R}_{-} \rightarrow \mathbb{R}^{d}$ with compact support $[-C, 0]$ which is continuous on $[-C, 0]$ but with a discontinuity in $-C$ is in $\mathcal{B}$. For such a function $\varphi$ which is firstly assumed to be positive, we define continuous functions $\vartheta_{n}$ and $\theta_{n}$ which equal $\varphi$ on $\left[-C+\frac{1}{n}, 0\right]$ and $[-C, 0]$, are linear on $\left[-C,-C+\frac{1}{n}\right]$ and $\left[-C-\frac{1}{n},-C\right]$ and are zero on $(-\infty,-C]$ and $\left(-\infty,-C-\frac{1}{n}\right]$, respectively, such that

$$
\vartheta_{n} \leqslant \varphi \leqslant \theta_{n} \quad \text { for every } n \in \mathbb{N} .
$$

Hence, every positive functional $\psi^{*} \in \mathcal{B}^{*}$ obeys the relations

$$
\left\langle\psi^{*}, \vartheta_{n}\right\rangle \leqslant\left\langle\psi^{*}, \varphi\right\rangle \leqslant\left\langle\psi^{*}, \theta_{n}\right\rangle .
$$

Since the functions $\vartheta_{n}$ are continuous and have compact support we obtain

$$
\left\langle\psi^{*}, \vartheta_{n}\right\rangle=\int d\left[\widetilde{\psi}^{*}(u)\right] \vartheta_{n}(u) \rightarrow \int d\left[\widetilde{\psi}^{*}(u)\right] \varphi(u) \quad \text { for } n \rightarrow \infty
$$


and analogously for $\theta_{n}$. Due to relation (5.26) we obtain the representation

$$
\left\langle\psi^{*}, \varphi\right\rangle=\int d\left[\widetilde{\psi}^{*}(u)\right] \varphi(u)
$$

for every positive functional $\psi^{*} \in \mathcal{B}^{*}$. The decompositions $\varphi=\varphi_{+}-\varphi_{-}$and $\psi^{*}=\psi_{+}^{*}-\psi_{-}^{*}$ show, that the representation (5.27) holds true for every continuous function $\varphi$ with support $[-C, 0]$ and with a possible discontinuity in $-C$ and every functional $\psi^{*} \in \mathcal{B}^{*}$.

If we consider the integrand of the covariance operator $R_{t}$ for $\varphi^{*}, \psi^{*} \in \mathcal{B}^{*}$ we obtain by (4.20) and (2.10)

$$
\begin{aligned}
\left\langle\psi^{*}, T^{* *}(s) \Upsilon^{*} \Upsilon T^{*}(s) \varphi^{*}\right\rangle & =y\left(-s, \widetilde{\psi}^{*}\right) y^{T}\left(-s, \widetilde{\varphi}^{*}\right) \\
& =\left(\int_{[-s, 0]} d\left[\widetilde{\psi}^{*}(u)\right] r_{s}(u)\right)\left(\int_{[-s, 0]} d\left[\widetilde{\varphi}^{*}(u)\right] r_{s}(u)\right)^{T}
\end{aligned}
$$

which entails by means of $(5.27)$

$$
\left\langle\psi^{*}, T^{* *}(s) \Upsilon^{*} \Upsilon T^{*}(s) \varphi^{*}\right\rangle=\sum_{k=1}^{d}\left\langle\psi^{*}, r_{s} e_{k}\right\rangle\left\langle\varphi^{*}, r_{s} e_{k}\right\rangle .
$$

On the other hand, for the operator $G: \mathbb{R}^{d} \rightarrow \mathcal{B}$ with $G(v):=v \mathbb{1}_{\{0\}}(\cdot)$ we have for $v \in \mathbb{R}^{d}$

$$
\left\langle G^{*} \psi^{*}, v\right\rangle_{\mathbb{R}^{d}}=\left\langle\psi^{*}, G(v)\right\rangle=\sum_{k=1}^{d}\left\langle\psi^{*}, e_{k} \mathbb{1}_{\{0\}}\right\rangle\left\langle e_{k}, v\right\rangle_{\mathbb{R}^{d}},
$$

which yields

$$
\begin{aligned}
\left\langle\psi^{*}, T(s) G G^{*} T^{*}(s) \varphi^{*}\right\rangle & =\left\langle G^{*} T^{*}(s) \psi^{*}, G^{*} T^{*}(s) \varphi^{*}\right\rangle_{\mathbb{R}^{d}} \\
& =\sum_{k=1}^{d}\left\langle T^{*}(s) \psi^{*}, e_{k} \mathbb{1}_{\{0\}}\right\rangle\left\langle e_{k}, G^{*} T^{*}(s) \varphi^{*}\right\rangle_{\mathbb{R}^{d}} \\
& =\sum_{k=1}^{d}\left\langle\psi^{*}, r_{s} e_{k}\right\rangle\left\langle\varphi^{*}, r_{s} e_{k}\right\rangle .
\end{aligned}
$$

It follows from (5.28) and (5.29) that $R_{t}$ coincides with the operator

$$
Q_{t}: \mathcal{B}^{*} \rightarrow \mathcal{B}, \quad Q_{t}=\int_{0}^{t} T(s) G G^{*} T^{*}(s) d s .
$$

which shows that the Gaussian Mehler semigroup $(P(t))_{t \geqslant 0}$ is an Ornstein-Uhlenbeck semigroup generated by $(T(t))_{t \geqslant 0}$ and $G$.

If the functions $\mathbb{1}_{\{0\}}(\cdot) e_{k}$ are elements of the space $\mathcal{B}$ we can define the linear, bounded operator

$$
G: \mathbb{R}^{d} \rightarrow \mathcal{B}, \quad G(v):=v \mathbb{1}_{\{0\}}(\cdot)
$$


As shown in the proof of Theorem 5.2 the covariance operator $R_{t}$ of $I(t)$ is then of the form

$$
R_{t} \varphi^{*}=\int_{0}^{t} T(s) G G^{*} T^{*}(s) \varphi^{*} d s .
$$

By means of the operator $G$ we can formulate the following Cauchy problem on the phase space $\mathcal{B}$ :

$$
\begin{aligned}
d Y(t) & =A Y(t) d t+G d W(t) \quad \text { for } t \geqslant 0 \\
Y(0) & =\varphi
\end{aligned}
$$

The operator $A$ is the generator of the semigroup $(T(t))_{t \geqslant 0}$ and $\varphi \in \mathcal{B}$. The Wiener process $W$ is the same as before with values in $\mathbb{R}^{d}$. See the Appendix for the definition of a weak solution of (5.31). The evolution equation (5.31) is of the form which is covered in the work [20] and [2] for evolution equations on Banach spaces.

Corollary 5.3. Let $\mathcal{B}$ be a Banach lattice which contains the functions $\mathbb{1}_{\{0\}}(\cdot) e_{k}$. Then there exists a unique weak solution $(Y(t, \varphi): t \geqslant 0)$ of $(5.31)$ which can be represented by

$$
Y(t, \varphi)=T(t) \varphi+\int_{0}^{t} T(t-s) G d W(s) \quad \text { for } t \geqslant 0,
$$

where the integral is a stochastic convolution integral introduced in Appendix C.

Proof. Since $R_{t}$ is the covariance operator of the Gaussian measure $I(t)$ on $\mathcal{B}$ and it is of the form (5.30) the result follows by Theorem C.5.

Corollary 5.4. Let $\mathcal{B}$ be a Banach lattice which contains the functions $\mathbb{1}_{\{0\}}(\cdot) e_{k}$. Then we have:

1) If $(X(t, \varphi): t \in \mathbb{R})$ is the solution of (1.3) in $\mathbb{R}^{d}$ then $\left(X_{t}(\cdot, \varphi): t \geqslant 0\right)$ is the weak solution of the Cauchy problem (5.31).

2) If $(Y(t, \varphi): t \geqslant 0)$ is the weak solution of the Cauchy problem (5.31) in $\mathcal{B}$ then the process $(Y(t, \varphi)(0): t \in \mathbb{R})$ with $Y(u, \varphi):=\varphi(u)$ for $u \leqslant 0$ is the solution of the differential equation (1.3) in $\mathbb{R}^{d}$.

Proof. The process $\left(X_{t}(\cdot, \varphi): t \geqslant 0\right)$ is a $\mathcal{B}$-valued adapted stochastic process by Theorem 3.1. Condition (A).4 guarantees that $t \mapsto\left\langle A^{*} \varphi^{*}, X_{t}\right\rangle$ is $P$-a.s. Lebesgue integrable for every $\varphi^{*} \in \operatorname{dom}\left(A^{*}\right)$.

It remains to prove that the segment process $\left(X_{t}(\cdot, \varphi): t \geqslant 0\right)$ obeys the variation of constants formula (5.32), which will follow from the coincidence of the stochastic integrals:

$$
\int_{0}^{t} T(t-s) G d W(s)=* \int_{0}^{t} T^{* *}(t-s) \Upsilon d W(s) \quad \text { P-a.s. }
$$


As in the proof of Theorem 5.2 one obtains for $v \in \mathbb{R}^{d}$ and $t \geqslant s \geqslant 0$ :

$$
\left\langle G^{*} T^{*}(t-s) \varphi^{*}, v\right\rangle_{\mathbb{R}^{d}}=\sum_{k=1}^{d}\left\langle\varphi^{*}, r_{t-s} e_{k}\right\rangle\left\langle e_{k}, v\right\rangle_{\mathbb{R}^{d}}
$$

and on the other hand

$$
\left\langle\Upsilon T^{*}(t-s) \varphi^{*}, v\right\rangle_{\mathbb{R}^{d}}=\sum_{k=1}^{d}\left\langle\varphi^{*}, r_{t-s} e_{k}\right\rangle\left\langle e_{k}, v\right\rangle_{\mathbb{R}^{d}} .
$$

Consequently, we have

$$
\begin{aligned}
\left\langle\varphi^{*}, \int_{0}^{t} T(t-s) G d W(s)\right\rangle & =\int_{0}^{t} G^{*} T^{*}(t-s) \varphi^{*} d W(s) \\
& =\int_{0}^{t} \Upsilon T^{*}(t-s) \varphi^{*} d W(s) \\
& =\int_{0}^{t}\left\langle T^{*}(t-s) \varphi^{*}, \Upsilon\right\rangle d W(s) \\
& =\left\langle\varphi^{*}, * \int_{0}^{t} T^{* *}(t-s) \Upsilon d W(s)\right\rangle
\end{aligned}
$$

which establishes the relation (5.33). Due to the uniqueness of the solutions the proof is complete.

Example 5.5. Let us finish with the Example 1.1 of the introduction, cf. also Example 2.3. The segment process of the solution of (1.1) is a Gaussian, strong Markov process on $C([\alpha, 0])$, its transition semigroup is represented in Example 4.5. Although the segment process is an Ornstein-Uhlenbeck process on the larger space $L_{\nu}^{2}$, it is not an Ornstein-Uhlenbeck process on $C([\alpha, 0])$ according to Theorem 5.2.

\section{Appendix}

\section{A Banach lattices}

Let $E$ be a real Banach space with a partial ordering $\leqslant$. If there exists an element $s \in E$ such that

$$
x \leqslant s, y \leqslant s \text { and }[x \leqslant z, y \leqslant z \Rightarrow s \leqslant z],
$$

then $s$ is called the least upper bound of $x$ and $y$ denoted by $s=\sup \{x, y\}$. In a similar way we define the greatest lower bound. The space $E$ is called a lattice if for each pair $(x, y)$ the least upper bound and the greatest lower bound exist. Moreover, $E$ is called vector lattice or Riesz space if the following conditions are satisfied for all $x, y, z \in E$ and $\alpha \geqslant 0$ :

$$
[x \leqslant y \Rightarrow x+z \leqslant y+z] \text { and }[x \leqslant y \Rightarrow \alpha x \leqslant \alpha y] .
$$


In a vector lattice $E$ we define $|x|:=\sup \{x,-x\}$. The Banach space $E$ is called a Banach lattice, if it satisfies

$$
|x| \leqslant|y| \Rightarrow\|x\| \leqslant\|y\| \quad \text { for all } x, y \in E .
$$

Note, that in a semi-normed vector lattice the positive cone $\{x \in E: x \geqslant 0\}$ is closed, see Proposition II.5.2 in [17]. The elements in the positive cone are called positive. Moreover, the dual space $E^{*}$ of the Banach lattice $E$ enjoys the property that for every element $x^{*} \in E^{*}$ there exist positive functionals $x_{+}^{*}, x_{-}^{*} \in E^{*}$ such that $x^{*}=x_{+}^{*}-x_{-}^{*}$, see Proposition II.5.5 in [17]. A functional $x^{*} \in E^{*}$ is called positive if $\left\langle x^{*}, x\right\rangle \geqslant 0$ for every positive $x \in E$.

\section{B Gaussian measures and semigroups}

Let $E$ denote a real separable Banach space and $E^{*}$ its dual space.

Definition B.1. Let $F$ be a subset of $E^{*}$. A subset $C \subseteq E$ is said to be a cylindrical set based on $F$ if it is of the form

$$
C=\left\{x \in E:\left(\left\langle x_{1}^{*}, x\right\rangle, \ldots,\left\langle x_{n}^{*}, x\right\rangle\right) \in B\right\}
$$

for some $n \in \mathbb{N}, x_{1}^{*}, \ldots, x_{n}^{*} \in F$ and a Borel set $B \in \mathfrak{B}\left(\mathbb{R}^{n}\right)$. Let $\mathcal{C}(E, F)$ denote the set of all cylindrical sets based on $F$ and $\mathcal{C}(E):=\mathcal{C}\left(E, E^{*}\right)$.

For every finite-dimensional $F \subseteq E^{*}$ the set $\mathcal{C}(E, F)$ is a $\sigma$-algebra and the set $\mathcal{C}(E)$ of all cylindrical sets is an algebra. We denote by $\sigma(\mathcal{C}(E))$ the $\sigma$-algebra induced by the algebra $\mathcal{C}(E)$. It is well known that $\sigma(\mathcal{C}(E))$ coincides with the Borel $\sigma$-algebra $\mathfrak{B}(E)$ since $E$ is separable.

Definition B.2. A function $\mu: \mathcal{C}(E) \rightarrow \mathbb{R}_{+}$with $\mu(E)=1$ is called a cylindrical measure on $\mathcal{C}(E)$ if for each finite subset $F \subseteq E^{*}$ the restriction $\left.\mu\right|_{\mathcal{C}(E, F)}$ is a probability measure.

\section{Definition B.3.}

(1) A probability measure $\mu$ on $\mathfrak{B}(\mathbb{R})$ is Gaussian if

(i) $\mu=\delta_{0}$, where $\delta_{0}$ is the Dirac measure,

or (ii) there exist $a \in \mathbb{R}$ and $b^{2}>0$ such that

$$
\mu(B)=\left(2 \pi b^{2}\right)^{-1 / 2} \int_{B} e^{-\frac{1}{2 b^{2}}(t-a)^{2}} d t \text { for every } B \in \mathfrak{B}(\mathbb{R}) .
$$

(2) a cylindrial measure $\mu$ on $\mathcal{C}(E, F)$ for $F \subseteq E^{*}$ is Gaussian if its onedimensional distributions $x^{*}(\mu)$ are Gaussian on $\mathbb{R}$ for every $x^{*} \in F$.

(3) a probability measure on $\mathfrak{B}(E)$ is Gaussian if it restricts to a Gaussian cylindrical measure on $\mathcal{C}(E)$.

For a cylindrical measure $\mu$ on $\mathcal{C}(E)$ the function

$$
\chi_{\mu}: E^{*} \rightarrow \mathbb{C}, \quad \chi_{\mu}\left(x^{*}\right):=\int_{X} e^{i\left\langle x^{*}, x\right\rangle} d \mu(x),
$$


is called characteristical functional or Fourier transform of the cylindrical measure $\mu$. The integral is the usual Lebesgue integral on the measure space $\left(E, \mathcal{C}\left(E,\left\{x^{*}\right\}\right), \mu\right)$ where $\mu$ is by definition a measure. The characteristical functional is positivedefinite, pseudo-continuous and $\chi_{\mu}(0)=1$. Conversely, every functional $\chi: E^{*} \rightarrow$ $\mathbb{C}$, which is positive-definite, pseudo-continuous and $\chi(0)=1$ is the characteristical function of a cylindrical measure on $\mathcal{C}(E)$. But in general that induced cylindrical measure is not a measure on $\sigma(\mathcal{C}(E))$.

Definition B.4. Let $H$ be a Hilbert space with norm $\|\cdot\|_{H}$. The cylindrical measure $\gamma_{H}$ on $\mathcal{C}(H)$ with the characteristical functional

$$
\chi_{\gamma_{H}}: H \rightarrow \mathbb{C}, \quad \chi_{\gamma_{H}}(h)=e^{-\frac{1}{2}\|h\|_{H}^{2}},
$$

is called the standard Gaussian cylindrical measure on $\mathrm{H}$.

Definition B.5. Let $H$ be a real Hilbert space and $U \in \mathcal{L}(H, E)$. Then $U$ is called $\gamma$-radonifying if the image cylindrical measure $U\left(\gamma_{H}\right)$ extends to a Gaussian Borel measure on $(E, \mathfrak{B}(E))$.

Remark B.6. The image cylindrical measure $U\left(\gamma_{H}\right)$ for $U \in \mathcal{L}(H, E)$ is a cylindrical Gaussian measure on $E$ whose characteristical functional $\chi$ is given by

$$
\chi\left(x^{*}\right)=\exp \left(-\frac{1}{2}\left\langle x^{*}, U U^{*} x^{*}\right\rangle\right) \quad \text { for all } x^{*} \in E^{*} .
$$

See Chapter VI in [18].

In [1] the concept of (generalized) Mehler semigroups is introduced which we present in the following definition in the Gaussian case. We denote by $B_{b}(E, \mathbb{R})$ the space of bounded Borel measurable real-valued functions on $E$.

Definition B.7. Let $(T(t))_{t \geqslant 0}$ be a $C_{0}$-semigroup on $E$ and $\left(\mu_{t}\right)_{t \geqslant 0}$ be a family of Gaussian measures on $\mathfrak{B}(E)$. If the measures satisfy

$$
\mu_{t+s}=T(s) \mu_{t} * \mu_{s} \quad \text { for all } s, t \geqslant 0,
$$

then $(P(t))_{t \geqslant 0}$ is called Gaussian Mehler semigroup defined by $\left(T(t), \mu_{t}\right)_{t \geqslant 0}$, where

$$
P(t): B_{b}(E, \mathbb{R}) \rightarrow B_{b}(E, \mathbb{R}), \quad P(t) f(x):=\int f(T(s) x+y) \mu_{t}(d y), \quad x \in E .
$$

\section{Remark B.8.}

The condition (2.34) on the measures is equivalent that the operators $(P(t))_{t \geqslant 0}$ form a semigroup, see [1]:

$$
P(t+s)=P(t) P(s) \quad \text { for every } s, t \geqslant 0 \quad \text { and } \quad P(0)=\mathrm{Id} .
$$

For an operator $Q \in \mathcal{L}(H, E)$ on a separable real Hilbert space $H$ we define for $t \geqslant 0$ :

$$
Q_{t}: E^{*} \rightarrow E, \quad Q_{t} x^{*}=\int_{0}^{t} T(s) Q Q^{*} T^{*}(s) x^{*} d s,
$$


where the integral is a Pettis integral defined by

$$
\left\langle y^{*}, Q_{t} x^{*}\right\rangle=\int_{0}^{t}\left\langle y^{*}, T(s) Q Q^{*} T^{*}(s) x^{*}\right\rangle d s \quad \text { for every } y^{*} \in E^{*} .
$$

In [2] it is shown that the operators $Q_{t}$ are $E$-valued and linear, bounded, positive and symmetric. Therefore, we can consider the reproducing kernel Hilbert space $H_{t}$ associated with $Q_{t}$ in the following way: on the range of $Q_{t}$ one defines an innner product by

$$
\left\langle Q_{t} x^{*}, Q_{t} y^{*}\right\rangle_{H_{t}}:=\left\langle x^{*}, Q_{t} y^{*}\right\rangle \text { for } x^{*}, y^{*} \in E^{*} .
$$

Let $H_{t}$ denote the Hilbert space completion of range $Q_{t}$ with respect to this inner product. Since $E$ is complete the inclusion mapping from range $Q_{t}$ into $E$ has a continuous extension to an injective linear map $j_{t}: H_{t} \rightarrow E$. By identifying $H_{t}$ with its dual space one obtains $Q_{t}=j_{t} j_{t}^{*}$.

Let $\gamma_{t}$ be the standard Gaussian cylindrical measure on $H_{t}$. If $j_{t}: H_{t} \rightarrow E$ is $\gamma$ radonifying for every $t \geqslant 0$ then the measures $\mu_{t}:=j_{t}\left(\gamma_{t}\right)$ are Gaussian measures on $\mathfrak{B}(E)$ with covariance operator $Q_{t}: E^{*} \rightarrow E$. Since the operators $Q_{t}$ obeys

$$
Q_{t+s}=T(s) Q_{t} T^{*}(s)+Q_{s},
$$

we have by use of the characteristical functionals that the measures $\mu_{t}$ satisfies the condition (2.34). Thus, $\left(T(t), \mu_{t}\right)_{t \geqslant 0}$ defines a Gaussian Mehler semigroup $(P(t))_{t \geqslant 0}$. But in addition to the general case in Definition B.7, the measures $\mu_{t}$ are closely related to the semigroup $(T(t))_{t \geqslant 0}$ by their covariance operator $Q_{t}$ which gives reason for the following definition:

Definition B.9. Let $(T(t))_{t \geqslant 0}$ be a $C_{0}$-semigroup on $E$ and $Q \in \mathcal{L}(H, E)$. If the embedding

$$
j_{t}: H_{t} \rightarrow E \quad \text { is } \gamma \text {-radonifying for every } t \geqslant 0,
$$

then $(P(t))_{t \geqslant 0}$ is called Ornstein-Uhlenbeck semigroup defined by $(T(t))_{t \geqslant 0}$ and $Q$ where

$$
P(t): B_{b}(E, \mathbb{R}) \rightarrow B_{b}(E, \mathbb{R}), \quad P(t) f(x):=\int f(T(s) x+y) \mu_{t}(d y), \quad x \in E .
$$

\section{Cauchy problem}

In this section we report shortly on results in [20] and [2] for the Cauchy problem on Banach spaces.

Let $E$ be a separable real Banach space, $E^{*}$ its dual space and $\mathfrak{B}(E)$ the Borel $\sigma$-algebra on $E$. We consider the following abstract Cauchy problem on $E$ :

$$
\begin{aligned}
d Y(t) & =A Y(t) d t+Q d W(t), \quad t \in[0, T], \\
Y(0) & =x_{0},
\end{aligned}
$$

for $x_{0} \in E$. Here $A$ is the generator of a strongly continuous semigroup $(T(t))_{t \geqslant 0}$ on $E$ and $Q$ is a bounded linear operator from a separable Hilbert space $\left(H,\langle\cdot, \cdot\rangle_{H}\right)$ into $E$. Denote by $(W(t): t \in[0, T])$ a cylindrical Wiener process with CameronMartin space $H$ according to the following definition: 
Definition C.1. A cylindrical Wiener process with Cameron-Martin space $H$ is a family $(W(t): t \in[0, T])$ of bounded linear operators from $H$ into $L_{P}^{2}(\Omega, \mathbb{R})$ with the following properties:

(1) for all $h \in H,(W(t) h: t \in[0, T])$ is a real-valued Brownian motion adapted to the filtration $\left\{\mathcal{F}_{t}\right\}_{t \in[0, T]}$.

(2) for all $s, t \in[0, T]$ and $h_{1}, h_{2} \in H$ we have

$$
\mathbb{E}\left[\left(W(s) h_{1}\right)\left(W(t) h_{2}\right)\right]=\min \{s, t\}\left\langle h_{1}, h_{2}\right\rangle_{H} .
$$

Example C.2. If $W$ is a standard Wiener process in $\mathbb{R}^{d}$, then

$$
W_{c}(t): \mathbb{R}^{d} \rightarrow L_{P}^{2}(\Omega, \mathbb{R}), \quad W_{c}(t) v:=\left\langle W_{c}(t), v\right\rangle_{\mathbb{R}^{d}}
$$

defines a cylindrical Wiener process $\left(W_{c}(t): t \geqslant 0\right)$ with Cameron-Martin space $\mathbb{R}^{d}$.

Definition C.3. An E-valued predictable process $\left(Y\left(t, x_{0}\right): t \in[0, T]\right)$ is called a weak solution of (3.36) if

(1) $s \mapsto\left\langle A^{*} x^{*}, Y\left(s, x_{0}\right)\right\rangle$ is P-a.s. integrable on $[0, T]$ for every $x^{*} \in \operatorname{dom}\left(A^{*}\right)$;

(2) for all $t \in[0, T]$ and $x^{*} \in \operatorname{dom}\left(A^{*}\right)$ we have

$$
\left\langle x^{*}, Y\left(t, x_{0}\right)\right\rangle=\left\langle x^{*}, x_{0}\right\rangle+\int_{0}^{t}\left\langle A^{*} x^{*}, Y\left(s, x_{0}\right)\right\rangle d s+\left(W(t)\left(Q^{*} x^{*}\right)\right) \quad P-a . s .
$$

In [2] it is shown that if the weak solution of (3.36) exists it can be represented by a variation of constants formula. Before we state the result we introduce the stochastic integration involved in this formula.

First, we introduce the straightforward definition of a stochastic integral for certain $H$-valued functions with respect to a cylindrical Wiener process with Cameron-Martin space $H$. For a step function $\mathbb{1}_{(a, b)} \otimes h$ with $h \in H$ define

$$
\int_{0}^{T} \mathbb{1}_{(a, b)}(s) \otimes h d W(s):=W(b) h-W(a) h .
$$

Extending this definition by linearity, we obtain a stochastic integral for $H$-valued step functions. Since for such a step function holds

$$
E\left(\int_{0}^{T} f(s) d W(s)\right)^{2}=\int_{0}^{T}\|f(s)\|_{H}^{2} d s
$$

this integral can be uniquely extended to the isometry

$$
J: L^{2}([0, T], H) \rightarrow L_{P}^{2}(\Omega, \mathbb{R}), \quad J f=\int_{0}^{T} f(s) d W(s) .
$$

Based on this integral for $H$-valued functions the following definition introduces an integral for certain $\mathcal{L}(H, E)$-valued operators: 
Definition C.4. Let $F:[0, T] \rightarrow \mathcal{L}(H, E)$ be a function, such that the map $t \mapsto F^{*}(t) x^{*}$ is strongly measurable and is an element of $L^{2}([0, T], H)$ for each $x^{*} \in E^{*}$. Then $F$ is called stochastically integrable w.r.t. $W$ if for all Borelmeasurable sets $A \subseteq(0, T)$ there exists a random variable $Y_{A} \in L_{P}^{2}(\Omega, E)$ such that for all $x^{*} \in E^{*}$ we have

$$
\left\langle x^{*}, Y_{A}\right\rangle=\int_{0}^{T} \mathbb{1}_{A}(s) F^{*}(s) x^{*} d W(s)
$$

P-almost surely. In this case one writes

$$
Y_{A}=\int_{A} F(s) d W(s)
$$

This definition of a stochastic integral enables the authors of [20] to derive the following result for the Cauchy problem (3.36):

Theorem C.5. The following assertions are equivalent:

(1) the Cauchy problem (3.36) has a weak solution $Y=\left(Y\left(t, x_{0}\right): t \in[0, T]\right)$;

(2) the function $t \mapsto T(t) Q$ is stochastically integrable w.r.t. $W$;

(3) The operator $V \in \mathcal{L}\left(E^{*}, E\right)$ defined by

$$
V x^{*}:=\int_{0}^{T} T(s) Q Q^{*} T^{*}(s) d s
$$

is a Gaussian covariance operator.

In this case the weak solution $Y$ is unique and obeys

$$
Y\left(t, x_{0}\right)=T(t) x_{0}+\int_{0}^{t} T(t-s) Q d W(s) \quad \text { for } t \in[0, T] .
$$

Moreover, for every $x^{*} \in \operatorname{dom}\left(A^{*}\right)$ the process $Y$ has a modification such that $\left\langle x^{*}, Y\left(t, x_{0}\right)\right\rangle$ is continuous.

By the variation of constants formula one derives easily in the next Corollary that the solution of the abstract Cauchy problem forms an Ornstein-Uhlenbeck process.

Corollary C.6. Let $(Y(t, x): t \geqslant 0)$ be the weak solution of (3.36) with $Y(0)=x$ for $x \in E$. Then we have for every $t \geqslant 0$

$$
\mathbb{E}\left[f(Y(t, x)]=P(t) f(x) \quad \text { for every } f \in B_{b}(E, \mathbb{R}),\right.
$$

where $(P(t))_{t \geqslant 0}$ is the Ornstein-Uhlenbeck semigroup defined by $(T(t))_{t \geqslant 0}$ and $Q$. 


\section{References}

[1] V. I. Bogachev, M. Röckner, and B. Schmuland. Generalized Mehler semigroups and applications. Probab. Theory Relat. Fields, 105(2):193-225, 1996.

[2] Z. Brzezniak and J.M.A.M. van Neerven. Stochastic convolution in separable Banach spaces and the stochastic linear Cauchy problem. Stud. Math., 143(1):43-74, 2000.

[3] A. Chojnowska Michalik. Representation theorem for general stochastic delay equations. Bull. Acad. Pol. Sci., Sr. Sci. Math. Astron. Phys., (26):635$642,1978$.

[4] B.D. Coleman and V.J. Mizel. Norms and semi-groups in the theory of fading memory. Arch. Ration. Mech. Anal., 23:87-123, 1966.

[5] J. B. Conway. A course in functional analysis. New York: Springer-Verlag, 1990.

[6] G. Da Prato and J. Zabczyk. Stochastic equations in infinite dimensions. Cambridge University Press, Cambridge, 1992.

[7] M.C. Delfour. The largest class of hereditary systems defining a $C_{0}$ semigroup on the product space. Can. J. Math., 32:969-978, 1980.

[8] M.C. Delfour and S.K. Mitter. Hereditary differential systems with constant delays. II: A class of affine systems and the adjoint problem. J. Differ. Equations, 18:18-28, 1975.

[9] E.B. Dynkin. Markov processes. Vol. I. Berlin: Springer-Verlag., 1965.

[10] R. E. Edwards. Functional analysis. Theory and applications. Holt Rinehart and Winston, New York, 1965.

[11] J. K. Hale and S. M. Verduyn Lunel. Introduction to functional differential equations. Springer New York, 1993.

[12] Y. Hino, S. Murakami, and T. Naito. Functional differential equations with infinite delay. Lecture Notes in Mathematics, 1473. Springer Berlin, 1991.

[13] A. Ichikawa. Generation of a semigroup on some product space with applications to evelution equations with delay. University of Warwick, Control Theory Centre, 52, 1976.

[14] N.N. Krasovskij. The approximation of a problem of analytic design of controls in a system with time-lag. J. Appl. Math. Mech., 28:876-885, 1964.

[15] V. J. Mizel and V. Trutzer. Stochastic hereditary equations: Existence and asymptotic stability. J. Integral Equations, 7:1-72, 1984.

[16] M. Riedle. Lyapunov exponents for linear delay equations in arbitrary phase spaces. Integral Equations Oper. Theory, to appear. 
[17] H. H. Schaefer. Banach lattices and positive operators. Berlin: SpringerVerlag, 1974.

[18] N.N. Vakhaniya, V.I. Tarieladze, and S.A. Chobanyan. Probability ddistributions on Banach spaces. Reidel Publishing Company, 1987.

[19] J.M.A.M. van Neerven and M. Riedle. Stochastic delay equations in the space of continuous functions. Institute of Mathematics, Humboldt University of Berlin, Preprint.

[20] J.M.A.M. van Neerven and L. Weis. Stochastic integration of functions with values in a Banach spaces. Acta Math., 166(2):131-170, 2005.

[21] R.B. Vinter and R.H. Kwong. The infinite time quadratic control problem for linear systems with state and control delays: an evolution equation approach. SIAM J. Control Optimization, 19:139-153, 1981. 